\title{
A UNIFIED APPROACH TO THE PAINLEVÉ TRANSCENDENTS
}

\author{
Norbert Steinmetz \\ Technische Universität Dortmund, Fakultät für Mathematik \\ D-44221 Dortmund, Germany; norbert.steinmetz@mathematik.uni-dortmund.de
}

\begin{abstract}
We utilise a recent approach via the so-called re-scaling method to derive a unified and comprehensive theory of the solutions to Painlevé's differential equations (I), (II) and (IV), with emphasis on the most elaborate equation (IV).
\end{abstract}

\section{Introduction}

Generally speaking, there are two different approaches to Painlevé's differential equations. The first one is based on the Riemann-Hilbert method and the method of isomonodromic deformations, and is strongly linked to the fields of special functions and mathematical physics, while the second one may be viewed as a part of complex analysis. We exemplarily mention the monographs by Fokas, Its, Kapaev, and Novokshënov [10] on one hand, and Gromak, Laine, and Shimomura [11] on the other. Although there is some overlap, the methods, issues, and results are quite different. It is even difficult to translate the results from one language into the other. The aim of this paper is to develop a unified theory for the solutions to the Painlevé equations

$$
\begin{array}{rlrl}
\text { (I) } \quad w^{\prime \prime} & =z+6 w^{2} \\
\text { (II) } & w^{\prime \prime} & =\alpha+z w+2 w^{3} \\
(\mathrm{IV}) & 2 w w^{\prime \prime} & =w^{\prime 2}+3 w^{4}+8 z w^{3}+4\left(z^{2}-\alpha\right) w^{2}+2 \beta
\end{array}
$$

with complex analytic methods. We shall investigate all equations simultaneously and in the same way, particular attention, however, will be payed to equation (IV), which usually is neglected in the literature. Several of the results in this paper have been proved in the past decade by different authors with different methods, in a bid to verify statements of the 'mathematical folklore', many of them dating back to Boutroux's papers $[4]^{1}$. Not every result stated and proved in the present paper is new. What is new is the methodical unification, which is based on the re-scaling method developed in [37] for equation (I), which itself was inspired by the so-called Zalcman re-scaling method [48, 49].

It is taken for granted that every solution is meromorphic on the plane. For recent proofs the reader is referred to Hinkkanen and Laine [15], Shimomura [31], and the author [36]. To the convenience of the reader we defer two major tools to Appendix A (Phragmén-Lindelöf principle) and Appendix B (existence of asymptotic expansions) at the end of the paper.

https://doi.org/10.5186/aasfm.2017.4203

2010 Mathematics Subject Classification: Primary 34M05, 30D35, 34M55, 30D45.

Key words: Stokes sector, Stokes ray, asymptotic expansion, re-scaling, pole-free sector, Airy equation, Weber-Hermite equation, sub-normal solution.

${ }^{1}$ There is some dissent about the validity of Boutroux's methods. 
Any transcendental solution to some Painlevé equation is called Painlevé transcendent ${ }^{2}$, more precisely, first, second, and fourth transcendent, respectively. Every Painlevé equation has a first integral $W$ :
(i) $w^{\prime 2}=4 w^{3}+2 z w-2 W$
$W^{\prime}=w$,
(ii) $w^{\prime 2}=w^{4}+z w^{2}+2 \alpha w-W$,
$W^{\prime}=w^{2}$,
(iv) $w^{\prime 2}=w^{4}+4 z w^{3}+4\left(z^{2}-\alpha\right) w^{2}-2 \beta-4 w W$,
$W^{\prime}=w^{2}+2 z w$.

At every pole $p$, the Painlevé transcendents and their first integrals have Laurent series developments

$$
\begin{aligned}
& \text { (i) } w=(z-p)^{-2}-\frac{p}{10}(z-p)^{2}-\frac{1}{6}(z-p)^{3}+\mathbf{h}(z-p)^{4}+\cdots \text {, } \\
& W=-(z-p)^{-1}-14 \mathbf{h}-\frac{p}{30}(z-p)^{3}-\frac{1}{24}(z-p)^{4}+\cdots, \\
& \text { (ii) } w=\epsilon(z-p)^{-1}-\epsilon \frac{p}{6}(z-p)-\frac{\alpha+\epsilon}{4}(z-p)^{2}+\mathbf{h}(z-p)^{3}+\cdots \text {, } \\
& W=-(z-p)^{-1}+10 \epsilon \mathbf{h}-\frac{7}{36} p^{2}-\frac{p}{3}(z-p)-\frac{1+\epsilon \alpha}{4}(z-p)^{2}+\cdots, \\
& \text { (iv) } w=\epsilon(z-p)^{-1}-p+\frac{\epsilon}{3}\left(p^{2}+2 \alpha-4 \epsilon\right)(z-p)+\mathbf{h}(z-p)^{2}+\cdots \text {, } \\
& W=-(z-p)^{-1}+2 \mathbf{h}+2(\alpha-\epsilon) p+\frac{1}{3}\left(4 \alpha-p^{2}-2 \epsilon\right)(z-p)+\cdots,
\end{aligned}
$$

respectively $(\epsilon= \pm 1)$; the coefficient $\mathbf{h}=\mathbf{h}(p)$ remains undetermined, and free: the pole $p$, the sign $\epsilon$, and $\mathbf{h}$ may be prescribed to define a unique solution in the same way than initial values $w_{0}$ and $w_{0}^{\prime}$ at $z_{0}$ do. The significance of $\mathbf{h}$ cannot be overestimated.

\section{Value distribution}

According to Boutroux [4] the Painlevé transcendents have order of growth at most 5/2, 3, and 4, respectively. These estimates were confirmed independently and with different methods by Shimomura [32] and the author [37]. In any case we have $m(r, w)=O(\log r)$, thus the growth of the Nevanlinna characteristic $T(r, w)$ is governed by the counting functions of poles $N(r, w)$ and $n(r, w)$. For notation and results in Nevanlinna theory the reader is referred to Hayman's monograph [13].

2.1. The key estimates. Analysing section 5.2 in Shimomura's paper [32] or section 6 in the author's paper [37] yields the following

Proposition 2.1. Let $w$ be any fourth transcendent, denote by $\mathcal{P}$ the set of non-zero poles of $w$, and set

$$
\triangle_{\delta}(p)=\left\{z:|z-p|<\delta|p|^{-1}\right\} \quad \text { and } \quad \mathcal{P}_{\delta}=\bigcup_{p \in \mathcal{P}} \triangle_{\delta}(p) .
$$

Then for $\delta>0$ sufficiently small, the discs $\triangle_{\delta}(p)$ are mutually disjoint and

$$
w=O(|z|) \quad\left(z \rightarrow \infty, z \notin \mathcal{P}_{\delta}\right) .
$$

This leads easily to the following estimates.

Proposition 2.2. For any fourth transcendent it is true that

$$
\begin{aligned}
& w^{\prime}=O\left(|z|^{2}\right) \text { and } W=O\left(|z|^{3}\right) \quad\left(z \rightarrow \infty, z \notin \mathcal{P}_{\delta}\right), \\
& w^{\prime}=O\left(|z|^{2}+|w|^{2}\right) \quad(z \rightarrow \infty \text { without restriction }) .
\end{aligned}
$$

\footnotetext{
${ }^{2}$ The original meaning of transcendent was different, namely: The solutions are transcendental functions of the 'two constants of integration'.
} 
Proof. The estimate of $w^{\prime}$ and $W$ in (5) follows from Cauchy's integral theorem for the first derivative applied to the circle $|\zeta-z|=\frac{\delta}{2}|z|^{-1}$, and $W^{\prime}=w^{2}+2 z w$, respectively, on combination with (4). To prove the last assertion, we note that $f(z)=\epsilon w^{\prime}(z)+w(z)^{2}+2 z w(z)$ is regular at any pole $p$ with residue $\epsilon$, and satisfies $f(z)=O\left(|z|^{2}\right)$ on $\partial \triangle_{\delta}(p)$, hence also on $\triangle_{\delta}(p)$ by the maximum principle. This yields $\left|w^{\prime}\right| \leq O\left(|z|^{2}\right)+|w|^{2}+|z w|=O\left(|z|^{2}\right)+O\left(|w|^{2}\right)$.

Remark 2.1. The data for the first and second transcendents reads as follows:

$$
\begin{aligned}
\triangle_{\delta}(p) & =\left\{z:|z-p|<\delta|p|^{-\frac{1}{4}}\right\}, \\
w & =O\left(|z|^{\frac{1}{2}}\right) \quad\left(z \rightarrow \infty, z \notin \mathcal{P}_{\delta}\right), \\
w^{\prime} & =O\left(|z|^{\frac{3}{4}}\right) \text { and } W=O\left(|z|^{\frac{3}{2}}\right) \quad\left(z \rightarrow \infty, z \notin \mathcal{P}_{\delta}\right), \\
w^{\prime} & =O\left(|z|^{\frac{3}{4}}+|w|^{\frac{3}{2}}\right) \quad(z \rightarrow \infty \text { without restriction }),
\end{aligned}
$$

and

$$
\begin{aligned}
\triangle_{\delta}(p) & =\left\{z:|z-p|<\delta|p|^{-\frac{1}{2}}\right\}, \\
w & =O\left(|z|^{\frac{1}{2}}\right) \quad\left(z \rightarrow \infty, z \notin \mathcal{P}_{\delta}\right), \\
w^{\prime} & =O(|z|) \text { and } W=O\left(|z|^{2}\right) \quad\left(z \rightarrow \infty, z \notin \mathcal{P}_{\delta}\right), \\
w^{\prime} & =O\left(|z|+|w|^{2}\right) \quad(z \rightarrow \infty \text { without restriction }) .
\end{aligned}
$$

2.2. The spherical derivative. The value distribution of any meromorphic function takes place in regions where the spherical derivative

$$
f^{\sharp}(z)=\frac{\left|f^{\prime}(z)\right|}{1+|f(z)|^{2}}
$$

is large, while $f$ behaves tame where $f^{\sharp}$ is small.

Proposition 2.3. Let $w$ be any fourth transcendent. Then $f(z)=w(z) / z$ has spherical derivative

$$
f^{\sharp}(z)=O(|z|) .
$$

Similarly, for any first and second transcendent, $f(z)=w(z)^{2} / z$ has spherical derivative $f^{\sharp}(z)=O\left(|z|^{\frac{1}{4}}\right)$ and $f^{\sharp}(z)=O\left(|z|^{\frac{1}{2}}\right)$, respectively.

Proof. In the first case we obtain from (6)

$$
f^{\sharp}(z) \leq \frac{\left|z w^{\prime}\right|}{|z|^{2}+|w|^{2}}+\frac{|w|}{|z|^{2}+|w|^{2}}=O(|z|)+O(1) .
$$

In the same manner $\left(6^{\prime}\right)$ gives

$$
\begin{aligned}
& \frac{\left|f^{\prime}(z)\right|}{1+|f(z)|^{\frac{3}{2}}} \leq \frac{2\left|w w^{\prime}\right|}{|z|\left(1+\left|w^{2} / z\right|^{\frac{3}{2}}\right)}+\frac{|w|^{2}}{|z|^{2}\left(1+\left|w^{2} / z\right|^{\frac{3}{2}}\right)} \\
& =|z|^{\frac{1}{4}} \frac{\left|w^{\prime}\right|}{|z|^{\frac{3}{4}}+|w|^{\frac{3}{2}}} \frac{2|z|^{\frac{1}{4}}|w|\left(|z|^{\frac{3}{4}}+|w|^{\frac{3}{2}}\right)}{|z|^{\frac{3}{2}}+|w|^{3}}+O(1)=O\left(|z|^{\frac{1}{4}}\right)
\end{aligned}
$$


(the term $\frac{2 x y^{2}\left(x^{3}+y^{3}\right)}{x^{6}+y^{6}}=\frac{2 t^{2}\left(1+t^{3}\right)}{1+t^{6}}$ with $x=|z|^{\frac{1}{4}}>0, y=|w|^{\frac{1}{2}}>0$ and $t=y / x>0$ is bounded), a fortiori $f^{\sharp}(z)=O\left(|z|^{\frac{1}{4}}\right)$. Similarly, $\left(6^{\prime \prime}\right)$ yields

$$
\begin{aligned}
f^{\sharp}(z) & \leq \frac{2\left|w w^{\prime}\right|}{|z|\left(1+\left|w^{2} / z\right|^{2}\right)}+\frac{|w|^{2}}{|z|^{2}\left(1+\left|w^{2} / z\right|^{2}\right)} \\
& =|z|^{\frac{1}{2}} \frac{\left|w^{\prime}\right|}{|z|+|w|^{2}} \frac{2|z|^{\frac{1}{2}}|w|\left(|z|+|w|^{2}\right)}{|z|^{2}+|w|^{4}}+O(1)=O\left(|z|^{\frac{1}{2}}\right) .
\end{aligned}
$$

2.3. The order of growth. There are several possibilities to prove

$$
T(r, w)=O\left(r^{\frac{5}{2}}\right), \quad T(r, w)=O\left(r^{3}\right), \text { and } T(r, w)=O\left(r^{4}\right)
$$

for first, second, and fourth transcendents, respectively:

a. Polar statistics. The fact that the discs $\triangle_{\delta}(p)$ are mutually disjoint and have area $\pi \delta^{2}|p|^{-1 / 2}, \pi \delta^{2}|p|^{-1}$, and $\pi \delta^{2}|p|^{-2}$ implies $n(r, w) r^{-\frac{1}{2}}=O\left(r^{2}\right)$, $n(r, w) r^{-1}=O\left(r^{2}\right)$, and $n(r, w) r^{-2}=O\left(r^{2}\right)$, respectively, hence the assertion follows from $m(r, w)=O(\log r)$ in any case.

b. Residue Theorem. It is not hard to construct a curve $\Gamma_{r}$ of length $O(r)$ which encloses exactly the poles of $w$ with $|p| \leq r$ and such that $W=O\left(|z|^{\frac{3}{2}}\right)$, $W=O\left(|z|^{2}\right)$, and $W=O\left(|z|^{3}\right)$, respectively, holds on $\Gamma_{r}$ : starting with the circle $C_{r}:|z|=r$, we replace any sub-arc $C_{r} \cap \triangle_{\delta}(p)$ by a sub-arc of $\partial \triangle_{\delta}(p)-$ the part outside $|z|=r$ if $|p| \leq r$, and the part inside $|z|=r$ otherwise. Then the estimate for $n(r, w)$ given in a. follows from

$$
\left.n(r, w)=-\frac{1}{2 \pi i} \int_{\Gamma_{r}} W(z) d z \quad \underset{p}{\operatorname{ras}} W=-1\right) .
$$

c. The Ahlfors-Shimizu characteristic of $f$ is given by

$$
T(r, f)=\frac{1}{\pi} \int_{0}^{r} A(t, f) \frac{d t}{t} \quad \text { with } A(t, f)=\int_{|z|<t} f^{\sharp}(z)^{2} d(x, y) .
$$

From Proposition 2.3 it follows that $A(t, f)=O\left(t^{2 \lambda+2}\right)$ holds, with $\lambda=\frac{1}{4}$, $\lambda=\frac{1}{2}$, and $\lambda=1$, respectively, hence also $T(r, f)=O\left(r^{2 \lambda+2}\right)$ and $T(r, w)=$ $O\left(r^{2 \lambda+2}\right)$. We note, however, that it is impossible to derive sharp bounds for $T(r, w)$ solely from the sharp estimates $w^{\sharp}=O\left(|z|^{\frac{3}{4}}\right), w^{\sharp}=O\left(|z|^{\frac{3}{2}}\right)$, and $w^{\sharp}=$ $O\left(|z|^{2}\right)$, respectively, which just lead to $T(r, w)=O\left(r^{\frac{7}{2}}\right), T(r, w)=O\left(r^{5}\right)$, and $T(r, w)=O\left(r^{6}\right)$. The reason for this is that $w^{\sharp}(z)$ is much too large on small neighbourhoods of the zeros of $w$, while the density $f^{\sharp}(z)^{2}$ of $A(t, f)$ is uniformly distributed but not too large.

d. For any transcendent, the entire function $F(z)=e^{-\int W(z) d z}$ has simple zeros at the poles of $w$ and satisfies $\log ^{+}|F(z)|=O\left(|z|^{\varrho}\right)$ as $z \rightarrow \infty$ outside $\mathcal{P}_{\delta}$ ( $\varrho=\frac{5}{2}, 3,4$, respectively), and this also holds inside the discs $\triangle_{\delta}(p)$ by the maximum principle. This implies $T(r, F)=m(r, F)=O\left(r^{\varrho}\right)$ and $T(r, W)=$ $T\left(r, F^{\prime} / F\right)=O\left(r^{\varrho}\right)$.

Remark 2.2. In accordance with Shimomura [32, p. 259], we note that the proofs of the estimates for $n(r, w)$ in Hille [14, p. 443], and also in Kitaev [21, p. 134] (with reference to Hille), are incorrect; both 'proofs' implicitly make use of the key fact that $w(z)^{-3} W(z)$ in case (I) and $w(z)^{-4} W(z)$ in case (II) (denoted $J(z)$ in $[14,21]$ ) is bounded on $\left\{z:|w(z)|>|z|^{1 / 2}\right\}$, which is more or less equivalent to what has to be proved. Several other attempts like $[27,45]$, working solely with $w^{\sharp}$, failed by the reason outlined in $\mathbf{c}$. 


\section{Painlevé transcendents and Yosida functions}

3.1. Re-scaling. Let $a$ and $b>-1$ be real parameters. The class $\tilde{\mathfrak{Y}}_{a, b}$ consists of all meromorphic functions $f$ such that the family $\left(f_{h}\right)_{|h|>1}$ of functions

$$
f_{h}(\mathfrak{z})=h^{-a} f\left(h+h^{-b} \mathfrak{z}\right)
$$

is normal on $\mathbf{C}$ in the sense of Montel, and all limit functions $\mathfrak{f}=\lim _{h_{n} \rightarrow \infty} f_{h_{n}}$ are $\not \equiv \infty$, at least one of them being non-constant. If, in addition, all limit functions are non-constant, then $f$ is said to belong to the Yosida class $\mathfrak{Y}_{a, b}$. The functions of class $\mathfrak{Y}_{0,0}$ were introduced by Yosida [47], and for arbitrary real parameters by the author [39]. The class $\mathfrak{Y}_{0,0}$ is universal in the sense that it contains all limit functions $\mathfrak{f}=\lim _{h_{n} \rightarrow \infty} f_{h_{n}}$ for $f \in \mathfrak{Y}_{a, b}$. The functions $f \in \tilde{\mathfrak{Y}}_{a, b}$ have striking properties, for example they satisfy $f^{\sharp}(z)=O\left(|z|^{|a|+b}\right), T(r, f)=O\left(r^{2+2 b}\right)$ and $m(r, f)=O(\log r)$, and even $T(r, f) \asymp r^{2+2 b}$ and $m\left(r, 1 / f^{\prime}\right)=O(\log r)$ if $f \in \mathfrak{Y}_{a, b}$.

Lemma 3.1. Normality of any re-scaled family $\left(f_{h}\right)_{|h|>1}$ is equivalent to

$$
\limsup _{z \rightarrow \infty} \frac{\left|f^{\prime}(z)\right||z|^{a-b}}{|z|^{2 a}+|f(z)|^{2}}<\infty
$$

while $f \in \mathfrak{Y}_{a, b}$ is equivalent to

$$
\liminf _{h \rightarrow \infty} \sup _{|z-h|<\delta|h|^{-b}} \frac{\left|f^{\prime}(z)\right||z|^{a-b}}{|z|^{2 a}+|f(z)|^{2}}>0
$$

for some (every) $\delta>0$, together with (9).

Proof. To prove the necessity of (9) we just note that $f_{h}^{\sharp}(0)=\frac{|h|^{-b-a}\left|f^{\prime}(h)\right|}{1+|h|^{-2 a}|f(h)|^{2}}$ has to be bounded on $|h|>1$ by Marty's criterion. Conversely, if $R>0$ is arbitrary, $|\mathfrak{z}|<R$ and $z=h+h^{-b} \mathfrak{z}=h\left(1+O\left(|h|^{-1-b}\right)\right)$, we obtain

$$
\limsup _{h \rightarrow \infty} f_{h}^{\sharp}(\mathfrak{z})=\limsup _{h \rightarrow \infty} \frac{|h|^{-b-a}\left|f^{\prime}(z)\right|}{1+|h|^{-2 a}|f(z)|^{2}}=\limsup _{z \rightarrow \infty} \frac{|z|^{-b-a}\left|f^{\prime}(z)\right|}{1+|z|^{-2 a}|f(z)|^{2}},
$$

uniformly with respect to $\mathfrak{z}$, hence $f_{h}^{\sharp}(\mathfrak{z})$ is bounded on $|\mathfrak{z}|<R,|h|>1$, and normality of $\left(f_{h}\right)_{|h|>1}$ follows from Marty's criterion. Finally, condition (10) is equivalent to the fact that every limit function $\mathfrak{f}=\lim _{h_{n} \rightarrow \infty} f_{h_{n}}$ is non-constant.

We note that by $\left(6^{\prime}\right),\left(6^{\prime \prime}\right)$, and $(6)$ the families $\left(w_{h}\right)_{|h|>1}$ of functions

$$
h^{-\frac{1}{2}} w\left(h+h^{-\frac{1}{4}} \mathfrak{z}\right), \quad h^{-\frac{1}{2}} w\left(h+h^{-\frac{1}{2}} \mathfrak{z}\right), \quad \text { and } h^{-1} w\left(h+h^{-1} \mathfrak{z}\right)
$$

for first, second and fourth Painlevé transcendents $w$, respectively, are normal.

Theorem 3.1. The first, second, and fourth Painlevé transcendents belong to the Yosida classes $\tilde{\mathfrak{Y}}_{\frac{1}{2}, \frac{1}{4}}, \tilde{\mathfrak{Y}}_{\frac{1}{2}, \frac{1}{2}}$, and $\tilde{\mathfrak{Y}}_{1,1}$, while the corresponding functions $f$ in Proposition 2.3 belong to the classes $\tilde{\mathfrak{Y}}_{0, \frac{1}{4}}, \tilde{\mathfrak{Y}}_{0, \frac{1}{2}}$, and $\tilde{\mathfrak{Y}}_{0,1}$, respectively. Every limit function $\mathfrak{w}=\lim _{h_{n} \rightarrow \infty} w_{h_{n}}$ satisfies

$$
\begin{aligned}
& \mathfrak{w}^{\prime \prime}=6 \mathfrak{w}^{2}+1, \\
& \mathfrak{w}^{\prime 2}=4 \mathfrak{w}^{3}+2 \mathfrak{w}-2 \mathfrak{c}, \\
& \mathfrak{w}^{\prime \prime}=2 \mathfrak{w}^{3}+\mathfrak{w}, \\
& \mathfrak{w}^{\prime 2}=\mathfrak{w}^{4}+\mathfrak{w}^{2}-\mathfrak{c}, \\
& \text { (iv) } 2 \mathfrak{w} \mathfrak{w}^{\prime \prime}=\mathfrak{w}^{\prime 2}+3 \mathfrak{w}^{4}+8 \mathfrak{w}^{3}+4 \mathfrak{w}^{2} \text {, } \\
& \mathfrak{w}^{\prime \prime}=2 \mathfrak{w}^{3}+6 \mathfrak{w}^{2}+4 \mathfrak{w}-2 \mathfrak{c}, \\
& \mathfrak{w}^{\prime 2}=\mathfrak{w}^{4}+4 \mathfrak{w}^{3}+4 \mathfrak{w}^{2}-4 \mathfrak{c} \mathfrak{w},
\end{aligned}
$$


respectively, with $\mathfrak{c}=\mathfrak{c}\left(\left\{h_{n}\right\}, w\right)$ some constant.

Proof. Normality of the families $\left(w_{h}\right)$ has already been proved. Every limit function $\mathfrak{w}=\lim _{h_{n} \rightarrow \infty} w_{h_{n}}$ is $\not \equiv \infty$ since it has a pole at $\mathfrak{z}=0$ if $\lim _{n \rightarrow \infty}\left|h_{n}\right| \operatorname{dist}\left(h_{n}, \mathcal{P}\right)=$ 0 , and is bounded on $|\mathfrak{z}|<\delta$ if $\liminf _{n \rightarrow \infty}\left|h_{n}\right| \operatorname{dist}\left(h_{n}, \mathcal{P}\right)>0$; in particular it follows that $w$ belongs to the respective Yosida class. In case of equation (IV), $\mathfrak{w}$ satisfies $2 \mathfrak{w} \mathfrak{w}^{\prime \prime}=\mathfrak{w}^{\prime 2}+3 \mathfrak{w}^{4}+8 \mathfrak{w}^{3}+4 \mathfrak{w}^{2}$; differentiating this equation yields $2 \mathfrak{w} \mathfrak{w}^{\prime \prime \prime}=\left(12 \mathfrak{w}^{2}+24 \mathfrak{w}+8\right) \mathfrak{w} \mathfrak{w}^{\prime}$, hence either $\mathfrak{w}=0$ or else $\mathfrak{w}^{\prime \prime \prime}=\left(6 \mathfrak{w}^{2}+12 \mathfrak{w}+4\right) \mathfrak{w}^{\prime}$ and $\mathfrak{w}^{\prime \prime}=2 \mathfrak{w}^{3}+6 \mathfrak{w}^{2}+4 \mathfrak{w}-2 \mathfrak{c}$ otherwise; on combination with the first equation (11)(iv) this leads to $\mathfrak{w}^{\prime 2}=\mathfrak{w}^{4}+4 \mathfrak{w}^{3}+4 \mathfrak{w}^{2}-4 \mathfrak{c} \mathfrak{w}$. For first and second transcendents it is even easier to derive the corresponding differential equations. Also the assertion on $f$ follows immediately from Proposition 2.3 and (9).

3.2. Elliptic and trigonometric limit functions. Any limit function $\mathfrak{w}=$ $\lim _{h_{n} \rightarrow \infty} w_{h_{n}}$ satisfies some differential equation

$$
\mathfrak{w}^{\prime 2}=P(\mathfrak{w} ; \mathfrak{c})
$$

where $P$ is a polynomial of degree three or four that depends on a complex parameter c. Constant limit functions (not: 'constant solutions') may only occur under special circumstances, namely if (i) $6 \mathfrak{w}^{2}+1=2 \mathfrak{w}^{3}+\mathfrak{w}-\mathfrak{c}=0$, (ii) $2 \mathfrak{w}^{3}+\mathfrak{w}=\mathfrak{w}^{4}+\mathfrak{w}^{2}-\mathfrak{c}=0$, and (iv) $3 \mathfrak{w}^{4}+8 \mathfrak{w}^{3}+4 \mathfrak{w}^{2}=\mathfrak{w}^{3}+3 \mathfrak{w}^{2}+2 \mathfrak{w}-\mathfrak{c}=0$, hence

(i) $\mathfrak{w} \equiv \pm i / \sqrt{6} \quad$ if $\mathfrak{c}= \pm i \sqrt{2 / 27}$,

(ii) $\mathfrak{w} \equiv 0 \quad$ if $\mathfrak{c}=0$

$\mathfrak{w} \equiv \pm i / \sqrt{2}$ if $\mathfrak{c}=-1 / 4$,

(iv) $\mathfrak{w} \equiv 0,-2 \quad$ if $\mathfrak{c}=0$

$\mathfrak{w} \equiv-2 / 3 \quad$ if $\mathfrak{c}=-8 / 27$.

In the respective cases (11), $P$ has discriminant (i) $2+27 \mathfrak{c}^{2}$, (ii) $\mathfrak{c}(4 \mathfrak{c}+1)^{2}$, and (iv) $\mathfrak{c}^{3}(27 \mathfrak{c}+8)$, and the zeros of the discriminant correspond to the exceptional parameters noted in (13). It is not hard to integrate equation (11) in the exceptional cases; only (ii) and (iv), and actually only the periods $\omega$ will be of interest.

$$
\begin{aligned}
& \text { (ii) } \mathfrak{c}=0 \quad \mathfrak{w}= \pm 1 / \sinh \mathfrak{z} \quad \omega=2 \pi i, \\
& \mathfrak{c}=-1 / 4 \quad \mathfrak{w}= \pm \tan (\mathfrak{z} / \sqrt{2}) / \sqrt{2} \quad \omega=\pi \sqrt{2}, \\
& \text { (iv) } \mathfrak{c}=0 \quad \mathfrak{w}=2 e^{ \pm 2 \mathfrak{z}} /\left(1-e^{ \pm 2 \mathfrak{z}}\right) \quad \boldsymbol{\omega}=\pi i, \\
& \mathfrak{c}=-8 / 27 \quad \mathfrak{w}=8 /\left(9 \tan ^{2}(\mathfrak{z} / \sqrt{3})-3\right) \quad \omega=\pi \sqrt{3} .
\end{aligned}
$$

If $P$ has mutually distinct zeros, then the non-constant solutions to (12) are elliptic functions of elliptic order two. The corresponding lattice $\Lambda=\Lambda_{\mathfrak{c}}$ has a basis $\left(\omega_{1}, \omega_{2}\right)$ such that $\left|\omega_{1}\right| \leq\left|\omega_{2}\right| \leq\left|\omega_{1} \pm \omega_{2}\right| ;\left|\omega_{1}\right|$ and $\left|\omega_{2}\right|$ are uniquely determined and depend continuously on the parameter $\mathfrak{c}$. Also $\omega_{2}$ tends to infinity as $\mathfrak{c}$ tends to any exceptional parameter, while $\omega_{1}$ has a definite limit (namely $\omega$ in (14)).

3.3. The cluster set. The constants of integration in the respective equations (11) are closely related to the corresponding first integrals. Inserting $\mathfrak{w}=$ $\lim _{h_{n} \rightarrow \infty} w_{h_{n}}$ into equation (1) we obtain

$$
\begin{array}{ll}
\text { (i) } \quad \mathfrak{c}=\lim _{h_{n} \rightarrow \infty} h_{n}^{-3 / 2} W\left(h_{n}\right) & \left(\inf \left|h_{n}\right|^{1 / 4} \operatorname{dist}\left(h_{n}, \mathcal{P}\right)>0\right), \\
\text { (ii) } \quad \mathfrak{c}=\lim _{h_{n} \rightarrow \infty} h_{n}^{-2} W\left(h_{n}\right) & \left(\inf \left|h_{n}\right|^{1 / 2} \operatorname{dist}\left(h_{n}, \mathcal{P}\right)>0\right), \\
\text { (iv) } \quad \mathfrak{c}=\lim _{h_{n} \rightarrow \infty} h_{n}^{-3} W\left(h_{n}\right) & \left(\inf \left|h_{n}\right| \operatorname{dist}\left(h_{n}, \mathcal{P}\right)>0\right),
\end{array}
$$


respectively. For any Painlevé transcendent $w$ we denote by $\mathfrak{C}(w)$ the set of all respective limits $(15) ; \mathfrak{C}(w)$ is called the cluster set of $w$. We note that in case (IV), say, the family $\left(W_{h}\right)_{|h|>1}, W_{h}(\mathfrak{z})=h^{-3} W\left(h+h^{-1} \mathfrak{z}\right.$ ) is quasi-normal (for the definition see [5]): if $w_{h_{n}}$ tends to $\mathfrak{w}$, then $W_{h_{n}}$ tends to some constant $\mathfrak{c} \in \mathfrak{C}(w)$, locally uniformly on $\mathbf{C} \backslash\{$ poles of $\mathfrak{w}\}$.

Proposition 3.1. In all cases the cluster set is closed, bounded, and connected, and contains the respective limits

$$
\begin{aligned}
& \text { (i) } \lim _{p_{n} \rightarrow \infty}-14 \mathbf{h}\left(p_{n}\right) p_{n}^{-3 / 2}, \\
& \text { (ii) } \lim _{p_{n} \rightarrow \infty} 10 \epsilon_{n} \mathbf{h}\left(p_{n}\right) p_{n}^{-2}-7 / 36 \quad\left(\epsilon_{n}=\underset{p_{n}}{\operatorname{res} w),}\right. \\
& \text { (iv) } \lim _{p_{n} \rightarrow \infty} 2 \mathbf{h}\left(p_{n}\right) p_{n}^{-3},
\end{aligned}
$$

where $\left(p_{n}\right)$ denotes any suitably chosen sequence of poles of $w$.

Proof. Again we will restrict ourselves to equation (IV). For $\delta>0$ sufficiently small, the closed discs $\bar{\triangle}_{\delta}(p)$ about the poles $p \neq 0$ are mutually disjoint, hence the domain $D_{\delta}=\mathbf{C} \backslash \bigcup_{p \in \mathcal{P}} \bar{\triangle}_{\delta}(p)$ is locally path-connected at infinity: any two points $a, b \in D_{\delta}$ may be joined by a curve contained in $D_{\delta}$ of spherical length comparable to the spherical distance of $a$ and $b$. We denote the corresponding cluster set of $z^{-3} W(z)$ as $z \rightarrow \infty$ on $D_{\delta}$ by $\mathfrak{C}_{\delta}(w)$, and note that $\mathfrak{C}(w)=\bigcup_{\delta>0} \mathfrak{C}_{\delta}(w)$ and $\mathfrak{C}_{\delta_{1}}(w) \subset$ $\mathfrak{C}_{\delta_{2}}(w)$ if $\delta_{2}<\delta_{1}$. The cluster sets $\mathfrak{C}_{\delta}(w)$ are closed, bounded in $\mathbf{C}$ since $z^{-3} W(z)$ is uniformly bounded on $D_{\delta}$, and connected by the special property of $D_{\delta}$. It remains to show that $\mathfrak{C}_{\eta}(w) \subset \mathfrak{C}_{\delta}(w)$ holds for $\delta>\eta$ sufficiently small (such that the discs $\triangle_{\delta}(p)$ are mutually disjoint), and that the limits (16)(iv) belong to $\mathfrak{C}_{\delta}(w)$. If $\eta \leq$ $\left|h_{n}\right| \operatorname{dist}\left(h_{n}, \mathcal{P}\right)<\delta$ and $\operatorname{dist}\left(h_{n}, \mathcal{P}\right)=\left|h_{n}-p_{n}\right|$ holds for some $p_{n} \in \mathcal{P}$, and if $\mathfrak{w}=\lim _{h_{n} \rightarrow \infty} w_{h_{n}}$ exists, we replace $h_{n}$ by $p_{n}$ with the following effect: from $p_{n}=$ $h_{n}+h_{n}^{-1} \mathfrak{z}_{n}$ with $\left(\mathfrak{z}_{n}\right)$ bounded, hence $\mathfrak{z}_{n} \rightarrow \mathfrak{z}_{0}$ as we may assume, it follows that $p_{n}^{-1}=(1+o(1)) h_{n}^{-1}, w_{h_{n}}(\mathfrak{z})=(1+o(1)) w_{p_{n}}\left(\mathfrak{z}-\mathfrak{z}_{0}+o(1)\right)$, and

$$
\hat{\mathfrak{w}}(\mathfrak{z})=\lim _{p_{n} \rightarrow \infty} w_{p_{n}}(\mathfrak{z})=\mathfrak{w}\left(\mathfrak{z}+\mathfrak{z}_{0}\right) .
$$

Thus $\hat{\mathfrak{w}}$ and $\mathfrak{w}$ satisfy the the same differential equation, this showing that $\mathfrak{c}=$ $\lim _{h_{n} \rightarrow \infty} h_{n}^{-3} W\left(h_{n}\right) \in \mathfrak{C}_{\eta}(w)$ coincides with one of the limits (16)(iv). Finally, if we start with some sequence $\left(w_{p_{n}}\right)$ with $p_{n} \in \mathcal{P}$, we may as well consider $\left(w_{h_{n}}\right)$ with $\left|h_{n}\right|\left|h_{n}-p_{n}\right|=\delta$ without changing the constant of integration, this showing that the limit $\mathfrak{c}=\lim _{p_{n} \rightarrow \infty} 2 \mathbf{h}\left(p_{n}\right) p_{n}^{-3}$ actually belongs to $\mathfrak{C}_{\delta}(w)$. We also obtain $\mathfrak{C}_{\delta}(w)=\mathfrak{C}_{\eta}(w)$ by combining both arguments.

\section{4. 'Pole-free' sectors}

4.1. Re-scaling and 'pole-free' sectors. Let $f$ be meromorphic on some sector $S:\left|\arg z-\theta_{0}\right|<\eta$. Then $S$ is called 'pole-free' for $f$, if for every $\delta>0, f$ has only finitely many poles on $S_{\delta}:\left|\arg z-\theta_{0}\right|<\eta-\delta$. If $w$ is any fourth Painlevé transcendent with 'pole-free' sector $S$, then the re-scaling process for sequences $\left(h_{n}\right)$ in $S_{\delta}$ leads to constant limit functions $\mathfrak{w}=\lim _{h_{n} \rightarrow \infty} w_{h_{n}}$. More precisely, these constants are contained in $\{0,-2,-2 / 3\}$ and also in the cluster set of $w / z$ restricted to $z \rightarrow \infty$ on $S$, which again is (compact) and connected. This proves that $w / z$ tends to one of the constants $0,-2$, and $-2 / 3$. The same argument works also for first and second transcendents, so that in any 'pole-free' sector of any Painlevé transcendent 
the following is true:

$$
\begin{array}{llll}
\text { (i) } & w=\sqrt{-z / 6}+o\left(|z|^{1 / 2}\right) & \text { and } & W(z)=\sqrt{-2 / 27} z^{3 / 2}+o\left(|z|^{3 / 2}\right), \\
\text { (ii) })_{a} & w=o\left(|z|^{1 / 2}\right) & \text { and } & W(z)=o\left(|z|^{2}\right), \\
\text { (ii) })_{b} & w=\sqrt{-z / 2}+o\left(|z|^{1 / 2}\right) & \text { and } & W(z)=-\frac{1}{4} z^{2}+o\left(|z|^{2}\right), \\
\text { (iv) })_{a} & w=-\frac{2}{3} z+o(|z|) & \text { and } & W(z)=-\frac{8}{27} z^{3}+o\left(|z|^{3}\right), \\
\text { (iv) })_{b} & w=-2 z+o(|z|) & \text { and } & W(z)=o\left(|z|^{3}\right), \\
\text { (iv) })_{c} & w=o(|z|) & \text { and } & W(z)=o\left(|z|^{3}\right),
\end{array}
$$

(for some branch of the square-root in (i) and (ii) $)_{b}$ ) as $z \rightarrow \infty$, uniformly on every closed sub-sector of $S$ in the respective cases.

4.2. Stokes sectors. The so-called Stokes sectors and corresponding Stokes rays
(i) $\quad \Sigma_{\nu}:|\arg z-2 \nu \pi / 5|<\pi / 5$,
$\sigma_{\nu}: \arg z=(2 \nu+1) \pi / 5$
(ii) ${ }_{a} \quad \Sigma_{\nu}:|\arg z-2 \nu \pi / 3|<\pi / 3$,
$\sigma_{\nu}: \arg z=(2 \nu+1) \pi / 3$,
(ii) ${ }_{b} \quad \Sigma_{\nu}:|\arg z-(2 \nu+1) \pi / 3|<\pi / 3$
$\sigma_{\nu}: \arg z=(2 \nu+2) \pi / 3$
(iv) ${ }_{a} \quad \Sigma_{\nu}:|\arg z-(2 \nu+1) \pi / 4|<\pi / 4$
$\sigma_{\nu}: \arg z=(\nu+1) \pi / 2$
(iv) $)_{b, c} \quad \Sigma_{\nu}:|\arg z-\nu \pi / 2|<\pi / 4$
$\sigma_{\nu}: \arg z=(2 \nu+1) \pi / 4$

will play an extraordinary role; note that $\sigma_{\nu}$ separates the adjacent Stokes sectors $\Sigma_{\nu}$ and $\Sigma_{\nu+1}$.

Proposition 4.1. Let $w$ be any Painlevé transcendent such that some of the corresponding asymptotics (17) holds as $z \rightarrow \infty$ on some single non-Stokes ray $\arg z=\bar{\theta}$. Then the Stokes sector which contains that ray is 'pole-free' for $w$.

Proof. Again our focus is on fourth transcendents $w$, and for definiteness we assume that the ray $\arg z=\bar{\theta}$ in question is contained in $\Sigma_{\nu}:|\arg z-\nu \pi / 2|<\pi / 4$, such that $w(z)=-2 z+o(|z|)$ holds as $z=r e^{i \bar{\theta}} \rightarrow \infty$. Then also $W^{\prime}=2 z w+w^{2}=$ $o\left(|z|^{2}\right)$ and $W(z)=o\left(|z|^{3}\right)$ hold as $z=r e^{i \bar{\theta}} \rightarrow \infty$, and the re-scaling method along any sequence $h_{n}=r_{n} e^{i \bar{\theta}}$ yields $\mathfrak{c}=0$ and limit functions satisfying

$$
\mathfrak{w}^{\prime 2}=\mathfrak{w}^{2}+4 \mathfrak{w}^{3}+4 \mathfrak{w}^{2}=\mathfrak{w}^{2}(\mathfrak{w}+2)^{2}, \quad \mathfrak{w}(0)=-2,
$$

hence $\mathfrak{w} \equiv-2$. Thus for $r \geq r_{0}$ there exist pole-free discs $\left|z-r e^{i \bar{\theta}}\right|<\rho(r) r^{-1}$ such that $\rho(r) \rightarrow \infty$ as $r \rightarrow \infty$. We define a sequence $\left(r_{n}\right)$ recursively by $r_{n+1}=r_{n}+8 r_{n}^{-1}$ and denote by $\theta_{n}\left(\bar{\theta}<\theta_{n} \leq \bar{\theta}+2 \pi\right)$ the largest angle such that $w$ has no poles on

$$
A_{n}=\left\{r e^{i \theta}: r_{n} \leq r \leq r_{n+1}, \bar{\theta} \leq \theta<\theta_{n}\right\} ;
$$

$r_{0}$ is chosen sufficiently large to ensure that $\theta_{0}<\bar{\theta}+2 \pi$. Let $J$ denote the set of integers $n$ such that $w$ has at least one pole $z_{n}$ on $\partial A_{n}$ (actually $\arg z_{n}=\theta_{n}$ ). Since $w$ is transcendental, $J$ is an infinite set, and re-scaling about any sequence $\left(z_{n_{\nu}}\right)$ with $n_{\nu} \in J$ then leads to one of the limit functions $\frac{2 e^{ \pm 2} s}{1-e^{ \pm 2}}$ with period $\pi i$ and poles $k \pi i$. This shows that, for $\nu \geq \nu_{0}$,

a. $w$ has poles $z_{n_{\nu}}+k(\pi i+o(1)) z_{n_{\nu}}^{-1}(-2 \leq k \leq 2)$ on $\left|z-z_{n_{\nu}}\right|<8\left|z_{n_{\nu}}\right|^{-1}$, and no others - note that $2 \pi<8<3 \pi$, and

b. the poles $z_{n_{\nu} \pm 1}$ also exist, that is, $n_{\nu} \pm 1 \in J$.

Thus $J$ contains all integers $n \geq n_{0}$, and the sequence $\left(z_{n}\right)_{n \geq n_{0}}$ is a sub-sequence of some sequence $\left(p_{k}\right)$ of poles satisfying

$$
p_{k+1}=p_{k}+(\omega+o(1)) p_{k}^{-1}
$$


with $\omega \in\{-\pi i, \pi i\}$ independent of $k$. By Lemma 4.1 below this implies

$$
\theta_{n}=\arg z_{n}=\arg p_{k_{n}} \rightarrow(2 \mu+1) \pi / 4
$$

for some $\mu \geq \nu$; in particular, the sector $\bar{\theta} \leq \arg z<(2 \nu+1) \pi / 4$ is 'pole-free' for $w$. In a similar way one can show that the sector $(2 \nu-1) \pi / 4<\arg z \leq \bar{\theta}$, hence $\Sigma_{\nu}$ is 'pole-free' for $w$. The proof in the other cases runs along the same lines.

4.3. Strings of poles. We will several times have to deal with sequences $\left(p_{k}\right)$ that tend to infinity and satisfy an approximative iterative scheme

$$
p_{k+1}=p_{k}+(\omega+o(1)) p_{k}^{-\tau},
$$

where $\omega \neq 0$ is complex and $\tau=s / t>-1$ is rational. Any such sequence is called a string.

Lemma 4.1. Any sequence $\mathfrak{p}=\left(p_{k}\right)$ satisfying the recursion (18) also satisfies

1. $p_{k}=(k(1+\tau) \omega)^{t /(s+t)}(1+o(1))$ and

2. $(s+t) \arg p_{k}=t \arg \omega+o(1) \bmod 2 \pi$, both times as $k \rightarrow \infty$, and

3. has counting function $n(r, \mathfrak{p})=\frac{r^{1+\tau}}{(1+\tau)|\omega|}(1+o(1))$ as $r \rightarrow \infty$.

Proof. Writing $q_{k}=p_{k}^{1+\tau}$ it follows from (18) that

$$
q_{k+1}=q_{k}\left(1+(\omega+o(1)) q_{k}^{-1}\right)^{1+\tau}=q_{k}+(\tau+1) \omega+o(1),
$$

hence 1. follows from $q_{k}=(\tau+1+o(1)) \omega k$, while assertions $\mathbf{2}$. and $\mathbf{3}$. are immediate consequences.

Remark 4.1. For $\tau$ some positive integer the analogy with the dynamics of the rational map $R(p)=p+\omega p^{-\tau}$ is evident (see [35] or any other text on rational dynamics); $R$ has a parabolic fixed point at infinity with $\tau+1$ invariant petals, and the iterates $R^{n}(p)$ converge to infinity asymptotically to the rays $(\tau+1) \arg p=\arg \omega$ $\bmod 2 \pi$.

Remark 4.2. It follows from the proof of Proposition 4.1 that 'pole-free' sectors are 'bordered' by strings of poles. In case (17)(ii) $)_{a}$ and (iv) $)_{a}$ the strings on both 'sides' have the interlacing property: 'between' any two poles with residue 1 there is one with residue -1 . Otherwise the poles on both strings have one and the same residue.

\section{Asymptotic expansions}

5.1. 'Pole-free' sectors and asymptotic expansions. A function $f$ that is meromorphic on some sector $S$ is said to have an asymptotic expansion

$$
f(z) \sim \sum_{\nu=m}^{\infty} a_{\nu} z^{-\nu / q} \quad \text { on } S,
$$

if $f(z)-\sum_{\nu=m}^{n} a_{\nu} z^{-\nu / q}=o\left(|z|^{-n / q}\right)$ holds for $n=m, m+1, \ldots$ as $z \rightarrow \infty$, uniformly on every proper sub-sector. It is obvious that $S$ is 'pole-free' if $f$ has an asymptotic expansion on $S$. For Painlevé transcendents the converse is also true.

Theorem 5.1. Every Painlevé transcendent with 'pole-free' sector $S$ has an asymptotic expansion on $S$.

Proof. Again we consider fourth transcendents only. Re-scaling along any sequence $\left(h_{n}\right)$ with $h_{n} \rightarrow \infty$ on any closed sub-sector $\tilde{S} \subset S$ yields limit functions without poles, hence constant limit functions either $\mathfrak{w}=0, \mathfrak{w}=-2$, or else $\mathfrak{w}=-2 / 3$. 
This leads to the 'leading terms' $o(|z|),-2 z+o(|z|)$, and $-2 z / 3+o(|z|)$ of the asymptotic expansions in question. We set $z v(z)=w(z)$ to obtain

$$
z^{-2}\left(v v^{\prime \prime}-v^{\prime 2}\right)=z^{-4}\left(2 \beta+v^{2}\right)-4 \alpha z^{-2} v^{2}+v^{2}(3 v+2)(v+2)
$$

with $c_{0}=\lim _{z \rightarrow \infty} v(z) \in\{0,-2,-2 / 3\}$. Theorem 12.1 in Appendix B applies if $c_{0}=-2$ and also if $c_{0}=-2 / 3$, yielding $w \sim z \sum_{k=0}^{\infty} c_{k} z^{-k}$ on $S$, but does not immediately apply if $c_{0}=0$. In the latter case we note that $w=o(|z|)$ implies $w^{\prime}=o(1)$ and $w^{\prime \prime}=o\left(|z|^{-1}\right)$, thus $4 z^{2} w^{2}$ dominates the terms $3 w^{4}, 8 z w^{3}$, and $-4 \alpha w^{2}$ in equation (IV) (they are $o\left(|z w|^{2}\right.$ ), and thus has to balance the term $-2 \beta+2 w w^{\prime \prime}-$ $w^{\prime 2}=-2 \beta+o(1)$, that is, $4 z^{2} w^{2}+2 \beta=o\left(|z|^{2}|w|^{2}\right)+o(1)$ holds and $z w$ tends to $\sqrt{-\beta / 2}=\gamma$. In that case we set $v(z)=z w(z)$ to obtain

$$
z^{-2}\left(v v^{\prime \prime}-v^{\prime 2}\right)=z^{-2}\left(4 \alpha v^{2}-8 v^{3}\right)+z^{-4}\left(v^{2}-3 v^{4}\right)+4 v^{2}+2 \beta .
$$

Then for $\gamma=\sqrt{-\beta / 2} \neq 0$ again Theorem 12.1 applies with $c_{0}=\lim _{z \rightarrow \infty} v(z)=\gamma$. Finally, for $\gamma=0$, we have $w=o\left(|z|^{-1}\right)$. If $w=o\left(|z|^{-n}\right)$ is assumed to be true for some $n \geq 1$ we obtain

$$
\begin{aligned}
4 z^{2} w^{2} & =2 w w^{\prime \prime}-w^{\prime 2}-3 w^{4}-8 z w^{3}+4 \alpha w^{2} \\
& =o\left(|z|^{-2 n-2}\right)+o\left(|z|^{-4 n}\right)+o\left(|z|^{-3 n+1}\right)+o\left(|z|^{-2 n}\right)=o\left(|z|^{-2 n}\right),
\end{aligned}
$$

hence $w=o\left(|z|^{-n-1}\right)$ and $w \sim 0$ (all coefficients vanish).

Remark 5.1. In case (I) and (II) we set $z=t^{2}$ and $t v(t)=w(z)$ to obtain

(i) $t^{-1} \ddot{v}+t^{-2} \dot{v}=t^{-3} v+4\left(1+6 v^{2}\right)$ and

(ii) $t^{-1} \ddot{v}+t^{-2} \dot{v}=4 \alpha t^{-3}+t^{-5} v+4 v\left(1+2 v^{2}\right)$,

respectively ( 'means differentiation with respect to $t$ ), with (i) $c_{0}=\lim _{t \rightarrow \infty} v(t)=$ $\sqrt{-1 / 6}$ on $S^{1 / 2}$ and (ii) $c_{0}=\sqrt{-1 / 2}$ or $c_{0}=0$; in case of $c_{0}=0$ we use $w=o\left(|z|^{1 / 2}\right)$ and $w^{3}=o(|z w|)$ to obtain $z w+\alpha=o(1)$.

5.2. Explicit expansions. It is not hard to confirm the following detailed expansions:

$$
\begin{array}{ll}
\text { (i) } & w(z)=\sqrt{-z / 6}-\frac{1}{48 z^{2}}+O\left(|z|^{-9 / 2}\right), \\
\text { (ii) })_{a} & w(z)=-\frac{\alpha}{z}+\frac{2 \alpha\left(\alpha^{2}-1\right)}{z^{4}}+O\left(|z|^{-7}\right), \\
\text { (ii) })_{b} & w(z)=\sqrt{-z / 2}+\frac{\alpha}{2 z}+O\left(|z|^{-5 / 2}\right), \\
\text { (iv) })_{a} & w(z)=-\frac{2}{3} z+\frac{\alpha}{z}-\frac{3 \alpha^{2}-9 \gamma^{2}+1}{4 z^{3}}+O\left(|z|^{-5}\right), \\
\text { (iv) })_{b} & w(z)=-2 z-\frac{\alpha}{z}+\frac{3 \alpha^{2}-\gamma^{2}+1}{4 z^{3}}+O\left(|z|^{-5}\right), \\
\text { (iv })_{c}^{ \pm} & w(z)= \pm \frac{\gamma}{z}-\frac{2 \gamma^{2} \mp \alpha \gamma}{2 z^{3}}+O\left(|z|^{-5}\right) \quad\left(\gamma^{2}=-\beta / 2\right) .
\end{array}
$$

5.3. The case $\boldsymbol{\beta}=\mathbf{0}$. The expansions (iv) $)_{c}^{ \pm}$are only significant if $\gamma=$ $\sqrt{-\beta / 2} \neq 0$. We thus suppose $\beta=0$ and $w \sim 0$ on some sector $S$, which means $w(z)=o\left(|z|^{-n}\right)$ as $z \rightarrow \infty$ on $S$ for every $n \in \mathbf{N}$. The logarithmic derivative $y=w^{\prime} / w$ satisfies

$$
y^{\prime}=P(z)-\frac{1}{2} y^{2} \quad \text { with } P(z)=2 z^{2}-2 \alpha+\frac{3}{2} z w(z)+2 w(z)^{2} \sim 2 z^{2}-2 \alpha .
$$


From the considerations in [41] it then follows that $y=w^{\prime} / w$ has an asymptotic expansion $y \sim \pm 2 z+\cdots$ on $S$, and $w \rightarrow 0$ on $S$ requirers $\operatorname{Re}\left( \pm z^{2}\right)<0$ on $S$ (for some sign), that is,

$$
\begin{aligned}
& (\mathrm{iv})_{c}^{-} \frac{w^{\prime}}{w} \sim-2 z+\frac{\alpha-1}{z}+\frac{\alpha^{2}-4 \alpha+3}{4 z^{3}}+\cdots \quad \text { if } S \subset \Sigma_{0} \cup \Sigma_{2}, \\
& (\mathrm{iv})_{c}^{+} \frac{w^{\prime}}{w} \sim 2 z-\frac{\alpha+1}{z}-\frac{\alpha^{2}+4 \alpha+3}{4 z^{3}}+\cdots \quad \text { if } S \subset \Sigma_{1} \cup \Sigma_{3} \text {. }
\end{aligned}
$$

A similar result holds in (19)(ii) $)_{a}$ if $\alpha=0: \frac{w^{\prime}}{w}= \pm \sqrt{z}-\frac{1}{4 z} \mp \frac{5}{32 z^{2} \sqrt{z}}+\cdots$ holds on sectors $\operatorname{Re}( \pm z \sqrt{z})<0$.

5.4. Asymptotics of the first integrals. From (1) and (19) it easily follows that

$$
\begin{aligned}
& \text { (i) } \quad W(z)=\frac{1}{9} \sqrt{-6} z^{3 / 2}+\frac{1}{48 z}+O\left(|z|^{-7 / 2}\right) \text {, } \\
& \text { (ii) })_{a} W(z)= \begin{cases}-\frac{\alpha^{2}}{z}+\frac{\alpha^{2}\left(\alpha^{2}-1\right)}{z^{4}}+O\left(|z|^{-7}\right) & (\alpha \neq 0), \\
O\left(|z|^{M}\right) e^{-\frac{2}{3}\left|\operatorname{Re} z^{\frac{3}{2}}\right|} & (\alpha=0),\end{cases} \\
& (\mathrm{ii})_{b} \quad W(z)=-\frac{1}{4} z^{2}+\sqrt{-2} \alpha z^{1 / 2}+\frac{1+4 \alpha^{2}}{8 z}+O\left(|z|^{-5 / 2}\right), \\
& \text { (iv) })_{a} W(z)=-\frac{8}{27} z^{3}+\frac{2}{3} \alpha z-\frac{3 \alpha^{2}+9 \gamma^{2}-1}{6 z}+O\left(|z|^{-3}\right) \text {, } \\
& (\text { iv) })_{b} W(z)=2 \alpha z+\frac{\alpha^{2}-\gamma^{2}+1}{2 z}+O\left(|z|^{-3}\right), \\
& (\mathrm{iv})_{c}^{ \pm} W(z)=\left\{\begin{array}{lr} 
\pm 2 \gamma z+\frac{\gamma^{2} \mp \alpha \gamma}{z^{z}}+O\left(|z|^{-3}\right) & (\gamma \neq 0) \\
O\left(|z|^{M}\right) e^{-\left|\operatorname{Re} z^{2}\right|} & (\gamma=0) .
\end{array}\right.
\end{aligned}
$$

5.5. The Hastings-McLeod solution. Equation $w^{\prime \prime}=z w+2 w^{3}$ has a unique solution, named after Hastings and McLeod [12], that decreases on the real line. Moreover, it satisfies $w(x) \sim 0$ as $x \rightarrow+\infty$ and $w(x) \sim \sqrt{-x / 2}$ as $x \rightarrow-\infty$. From Proposition 4.1 and Theorem 5.1 it follows that the asymptotic expansions $w \sim 0$ and $w(z) \sim \sqrt{-z / 2}+\cdots$ hold throughout $|\arg z|<\pi / 3$ and $|\arg z-\pi|<\pi / 3$, respectively. The poles are asymptotically restricted to the sectors $|\arg z \mp \pi / 2|<$ $\pi / 6$. Writing $w^{\prime \prime}=\left(z+2 w(z)^{2}\right) w$ and noting that $w(z)=o\left(|z|^{-n}\right)$ on $|\arg z|<\pi / 3$ for every $n \in \mathbf{N}$ yields $w(z) \sim k \operatorname{Ai}(z)$ for some real constant $k$, actually $k=1$; Ai denotes the Airy function. More general, for $0<k<1$ there exists a unique solution, named after Ablowitz and Segur [1, 2], that is bounded on $\mathbf{R}$ and satisfies $w(x) \sim k \operatorname{Ai}(x)$ as $x \rightarrow+\infty$. Since in that case $w$ has the asymptotics $w(x)=(-x)^{-1 / 4}$ times an oscillating term as $x \rightarrow-\infty, w$ cannot have an asymptotic expansion on some sector about $\arg z=\pi$, and hence the poles of $w$ must accumulate at $\arg z=\pi$. Also Proposition 4.1 is void in the present case since $\arg z=\bar{\theta}=\pi$ is a Stokes ray.

5.6. The Clarkson-McLeod solution. For $\beta=0$ and $\alpha$ real it is conjectured that there exists a unique real solution to equation (IV) - the so-called ClarksonMcLeod solution [6, 19] - satisfying $w(x) \sim 0$ as $x \rightarrow+\infty$ and $w(x) \sim-2 x+\cdots$ as $x \rightarrow-\infty$. If it exists, and existence is supported by numerical experiments and by analogy to the Hastings-McLeod solution, then the corresponding asymptotic expansions $w \sim 0$ (even $w^{\prime} / w \sim-2 z+\cdots$ by $(20)$ ) and $w \sim-2 z+\cdots$ hold on $|\arg z|<\pi / 4$ and $|\arg z-\pi|<\pi / 4$, respectively. 
5.7. Bäcklund transfomations. Generally speaking, a Bäcklund transformation is any change of variables that transforms some given differential equation into itself or into a differential equation of the same type. We confine ourselves to equation (IV). The Bäcklund transformation due to Lukashevich [22] (see also [11])

$$
\tilde{w}=\frac{w^{\prime}-2 \gamma-2 z w-w^{2}}{2 w} \quad\left(\gamma^{2}=-\beta / 2\right)
$$

transforms equation (IV) into the same equation with new parameters

$$
\tilde{\alpha}=(1-\alpha+3 \gamma) / 2 \text { and } \quad \tilde{\gamma}=(1+\alpha+\gamma) / 2 \text {; }
$$

the (pointwise) inverse transformation is given by

$$
w=-\frac{\tilde{w}^{\prime}+2 \tilde{\gamma}+2 z \tilde{w}+\tilde{w}^{2}}{2 \tilde{w}} \quad\left(\tilde{\gamma}^{2}=-\tilde{\beta} / 2\right)
$$

with $\alpha=(-1-\tilde{\alpha}+3 \tilde{\gamma}) / 2$ and $\gamma=(-1+\tilde{\alpha}+\tilde{\gamma}) / 2$; we note that

$$
\tilde{\alpha}-\tilde{\gamma}=-(\alpha-\gamma)
$$

holds in any case. Applying (22) twice yields equation (IV) with parameters $\alpha+1$ and $\gamma+1$. Lukashevich's transformations form the master pair (actually, quadruple: $\gamma$ and $\tilde{\gamma}$ may be replaced by $-\gamma$ and $-\tilde{\gamma}=(1+\alpha-\gamma) / 2$, respectively), since every (known and unknown) Bäcklund transformation may be expressed by certain compositions of (22) and (23), see [3].

5.8. Invariance. We close this section by proving the invariance of cluster sets, 'pole-free' sectors, asymptotic expansions and the Nevanlinna characteristic under Bäcklund transformations.

Proposition 5.1. For every fourth transcendent the cluster set, 'pole-free' sectors, and the Nevanlinna characteristic remain invariant under the corresponding Bäcklund transformations (22) and (23), the latter meaning $T(r, \tilde{w}) \asymp T(r, w)$. Moreover, the Bäcklund transformation (22) changes

$$
w \sim\left\{\begin{array} { l } 
{ - \frac { 2 } { 3 } z + \cdots } \\
{ - 2 z + \cdots }
\end{array} \quad \text { into } \tilde { w } \sim \left\{\begin{array}{l}
-\frac{2}{3} z+\cdots \\
\tilde{\gamma} / z+\cdots
\end{array}\right.\right.
$$

For $\gamma \neq 0$ it also changes

$$
w \sim\left\{\begin{array} { l } 
{ - \gamma / z + \cdots } \\
{ \gamma / z + \cdots }
\end{array} \quad \text { into } \tilde { w } \sim \left\{\begin{array}{l}
-\tilde{\gamma} / z+\cdots \\
-2 z+\cdots
\end{array}\right.\right.
$$

while for $\gamma=0, w \sim 0(w \not \equiv 0)$ is changed into

$$
\tilde{w} \sim \begin{cases}-2 z+\cdots & \text { on } \Sigma_{0} \text { and } \Sigma_{2}, \\ -\tilde{\gamma} / z+\cdots & \text { on } \Sigma_{1} \text { and } \Sigma_{3} .\end{cases}
$$

Remark 5.2. A similar result holds for second transcendents.

Proof. The assertion about the cluster set follows from

$$
w^{\prime}-2 \gamma-2 z w-w^{2}=2 w \tilde{w}=-\left(\tilde{w}^{\prime}+2 \tilde{\gamma}+2 z \tilde{w}+\tilde{w}^{2}\right),
$$

hence $w^{\prime}+\tilde{w}^{\prime}-2(\gamma-\tilde{\gamma})=2 z w+w^{2}-\left(2 z \tilde{w}+\tilde{w}^{2}\right)=W^{\prime}-\tilde{W}^{\prime}$ and

$$
W-\tilde{W}=w+\tilde{w}-2(\gamma-\tilde{\gamma}) z+\text { const. }=O(|z|) \quad\left(z \notin \mathcal{P}_{\delta}(w) \cup \mathcal{P}_{\delta}(\tilde{w})\right) .
$$

Moreover, from $\tilde{w}=\frac{w^{\prime}-2 \gamma}{2 w}-z-\frac{w}{2}$ it follows that

$$
T(r, \tilde{w})+O(\log r)=N(r, \tilde{w}) \leq N(r, 1 / w)+N(r, w) \leq 2 T(r, w)+O(1),
$$


and, in the same manner, $T(r, w) \leq 2 T(r, \tilde{w})+O(\log r)$. To prove the statements about the change of the asymptotic expansions is just a matter of elementary computations, at least if $\beta \neq 0$. For $\gamma=0$ it is also true that the expansion (iv) $b$ is transformed into (iv) $)_{c}^{+}$. The other cases need a more subtle argument. From (20), $\gamma=0, w \sim 0$, and $\tilde{w}=\frac{w^{\prime}}{2 w}-z-\frac{w}{2}$ we obtain $\tilde{w} \sim-2 z+\frac{\alpha-1}{2 z}+\cdots$ on $\Sigma_{0} \cup \Sigma_{2}$, and $\tilde{w} \sim-\frac{\alpha+1}{2 z}+\cdots$ on $\Sigma_{1} \cup \Sigma_{3}$.

\section{Truncated Painlevé transcendents}

6.1. Truncated solutions. Painlevé transcendents having some 'pole-free' sector $S:\left|\arg z-\theta_{\nu}\right|<\epsilon$ about the Stokes ray $\sigma_{\nu}: \arg z=\theta_{\nu}$ are called truncated (along $\left.\sigma_{\nu}\right)$. By Theorem 5.1, $w$ has an asymptotic expansion on $S$, and from Proposition 4.1 applied to $\theta_{\nu}-\epsilon<\bar{\theta}<\theta_{\nu}$ and $\theta_{\nu}<\bar{\theta}<\theta_{\nu}+\epsilon$ it follows that the asymptotic expansion extends to $\Sigma_{\nu} \cup \sigma_{\nu} \cup \Sigma_{\nu+1}$. In particular, any transcendent $w$ that is truncated along $\sigma_{\nu}$ has the same asymptotic expansion on adjacent Stokes sectors $\Sigma_{\nu}$ and $\Sigma_{\nu+1} \cdot\left({ }^{3}\right)$ The converse is also true:

Proposition 6.1. Let $w$ be any Painlevé transcendent that has the same asymptotic expansion (19) on adjacent Stokes sectors $\Sigma_{\nu}$ and $\Sigma_{\nu+1}$. Then $w$ is truncated along $\sigma_{\nu}$, and the asymptotic expansion holds on $\Sigma_{\nu} \cup \sigma_{\nu} \cup \Sigma_{\nu+1}$.

Proof. In any case $F(z)=e^{-\int W(z) d z}$ is an entire function of finite order with simple zeros at the poles of $w$. Since again the idea of proof is the same in all cases we will consider this time (19)(iv) $)_{b}$, say, with $\Sigma_{\nu}:|\arg z-\nu \pi / 2|<\pi / 4$ and

$$
W(z)=2 \alpha z+\frac{\alpha^{2}-\gamma^{2}+1}{2 z}+O\left(|z|^{-3}\right)
$$

which holds by (21), hence

$$
H(z)=F(z) e^{\alpha z^{2}} z^{\left(\alpha^{2}-\gamma^{2}+1\right) / 2}= \begin{cases}c_{\nu}+o(1) & \text { as } z \rightarrow \infty \text { on } \Sigma_{\nu}, \\ c_{\nu+1}+o(1) & \text { as } z \rightarrow \infty \text { on } \Sigma_{\nu+1}\end{cases}
$$

holds for some complex constants $c_{\nu}$ and $c_{\nu+1}$. We set

$$
f(\zeta)=H(z) \text { with } z=e^{i(2 \nu+1) \pi / 4} \sqrt{\zeta} \text { and } \sqrt{\zeta}>0 \text { on } \zeta>0 .
$$

Since $f$ has finite order $\lim \sup _{\zeta \rightarrow \infty} \frac{\log \log |f(\zeta)|}{\log |\zeta|} \leq 4$ on $|\arg \zeta|<\pi-\eta$ and limits $c_{ \pm}$as $\zeta=r e^{ \pm i \delta} \rightarrow \infty$ for every $0<\delta<\pi$, the Phragmén-Lindelöf Principle (see Theorem 11.1 in Appendix A) yields $c_{-}=c_{+}=c$ and $f(\zeta) \rightarrow c$ on $|\arg \zeta| \leq \delta$. From Proposition 4.1 it then follows that the half-plane $|\arg z-(2 \nu+1) \pi / 4|<\pi / 2$ is 'pole-free' for $w$, and $w$ has the asymptotic expansion (19)(iv) $b_{b}$ there.

6.2. Existence and uniqueness of truncated solutions. We will first prove a result valid in all cases, which will afterwards be refined for equation (I).

Theorem 6.1. ([25, 26] and many others) Given any appropriate Stokes ray $\sigma_{\nu}$, there exist

(i) first transcendents that are truncated along $\sigma_{\nu}$ and $\sigma_{\nu+1}$,

(ii) solutions to (II) that are truncated along $\sigma_{\nu}$, and

(iv) solutions to (IV) that are truncated along $\sigma_{\nu}$;

\footnotetext{
${ }^{3}$ In cases (i) and (ii) "the same expansion" means that the square-root $\sqrt{-z}$ is assumed to be continuous across $\sigma_{\nu}$.
} 
in (ii) and (iv) rational solutions are included. The asymptotic expansion (19) along $\sigma_{\nu}$, hence on $\Sigma_{\nu} \cup \sigma_{\nu} \cup \Sigma_{\nu+1}$ may be prescribed; it uniquely determines the solutions in question.

Proof. Again we restrict ourselves to fourth transcendents. By Theorem 12.2 in Appendix A there exists some fourth transcendent $w$ with prescribed asymptotics (iv) on any given sector $S$ of central angle $\pi / 2$. In particular, if $S$ contains some Stokes ray $\sigma_{\nu}$, the asymptotic expansion holds on $\Sigma_{\nu} \cup \sigma_{\nu} \cup \Sigma_{\nu+1}$ by Proposition 4.1, and $w$ is truncated along $\sigma_{\nu}$. The proof is similar in the other cases on combination of Theorem 12.2 and Proposition 4.1.

To prove uniqueness we consider fourth Painlevé transcendents $w_{1}$ and $w_{2}$ having the same asymptotic expansion (19) on some sector $S$. Then $u=w_{1}-w_{2}$ tends to zero as $z \rightarrow \infty$ more than geometrically fast on every proper sub-sector of $S$, that is, for every positive integer $n, z^{n} u$ tends to zero. In all cases we will derive a linear differential equation

$$
y^{\prime \prime}+Q(z) y=0,
$$

which on one hand has no non-trivial solution that tends to zero as $z \rightarrow \infty$ on $S$ if the sector $S$ has central angle $\Theta>\pi / 2$, and on the other hand has some non-trivial solution tending to zero provided $u=w_{1}-w_{2} \not \equiv 0$. This will show that $w_{1}=w_{2}$.

From

$$
\begin{aligned}
w_{1} w_{1}^{\prime \prime}-w_{2} w_{2}^{\prime \prime} & =w_{1} u^{\prime \prime}+w_{2}^{\prime \prime} u=w_{2} u^{\prime \prime}+w_{1}^{\prime \prime} u, \text { hence } \\
2 w_{1} w_{1}^{\prime \prime}-2 w_{2} w_{2}^{\prime \prime} & =\left(w_{1}+w_{2}\right) u^{\prime \prime}+\left(w_{1}^{\prime \prime}+w_{2}^{\prime \prime}\right) u, \\
w_{1}^{\prime 2}-w_{2}^{\prime 2} & =\left(w_{1}^{\prime}+w_{2}^{\prime}\right) u^{\prime}, \\
w_{1}^{4}-w_{2}^{4} & =\left(w_{1}^{3}+w_{1}^{2} w_{2}+w_{1} w_{2}^{2}+w_{2}^{3}\right) u \text { etc. }
\end{aligned}
$$

we obtain $\left(w_{1}+w_{2}\right) u^{\prime \prime}+\left(w_{1}^{\prime \prime}+w_{2}^{\prime \prime}\right) u=\left(w_{1}^{\prime}+w_{2}^{\prime}\right) u^{\prime}+h(z) u$ with

$$
h(z)=3\left(w_{1}^{3}+w_{1}^{2} w_{2}+w_{1} w_{2}^{2}+w_{2}^{3}\right)+8 z\left(w_{1}^{2}+w_{1} w_{2}+w_{2}^{2}\right)+4\left(z^{2}-\alpha\right)\left(w_{1}+w_{2}\right),
$$

hence the linear differential equation

$$
u^{\prime \prime}+a(z) u^{\prime}+b(z) u=0
$$

with coefficients $a(z)=-\frac{w_{1}^{\prime}(z)+w_{2}^{\prime}(z)}{w_{1}(z)+w_{2}(z)}$ and $b(z)=\frac{w_{1}^{\prime \prime}(z)+w_{2}^{\prime \prime}(z)-h(z)}{w_{1}(z)+w_{2}(z)}$. In case of $(19)(\mathrm{iv})_{c}$ we first assume in addition that $\gamma \neq 0$ (otherwise we would have to divide by $w_{1}+w_{2} \sim$ 0 which would cause difficulties). The substitution

$$
y=u e^{\frac{1}{2} \int a(z) d z}=\left(w_{1}(z)+w_{2}(z)\right)^{-1 / 2} u=z^{\epsilon / 2}(c+o(1)) u
$$

$(c \neq 0, \epsilon=1 \text { in case (iv) })_{c}^{ \pm}$, and $\epsilon=-1$ otherwise) transforms (27) into (26) with $Q(z)=b(z)-\frac{1}{4} a(z)^{2}-\frac{1}{2} a^{\prime}(z)=b(z)+O\left(|z|^{-2}\right)$, and, in more detail,

$$
Q(z)= \begin{cases}\frac{4}{3} z^{2}+O\left(|z|^{-2}\right) & (\mathrm{iv})_{a}, \\ -4 z^{2}-8 \alpha+O\left(|z|^{-2}\right) & (\mathrm{iv})_{b}, \\ -4 z^{2}+4 \alpha \mp 12 \gamma+O\left(|z|^{-2}\right) & (\mathrm{iv})_{c}^{ \pm} .\end{cases}
$$

The asymptotic integration of (26) is well understood (see Hille [14] or else Wasow [44]). We just need a qualitative result: writing $Q(z)=c z^{\tau}+o\left(|z|^{\tau}\right)$, every non-trivial solution to (26) tends to infinity exponentially on some sub-sector of $S$ if the central angle $\Theta$ of $S$ is greater than $\frac{2 \pi}{\tau+2}$. In our case this yields $y \equiv 0$ if $\Theta>\pi / 2$, and this proves Theorem 6.1 up to the case (iv) ${ }_{c}^{ \pm}$with $\gamma=0$. Here we compare the solution $w_{1}=w \sim 0$ with the trivial solution $w_{2} \equiv 0$. From $w(z)=o\left(|z|^{-n}\right)$ for every 
$n \in \mathbf{N}$, and $\left(w^{\prime}(z) / w(z)\right)^{2}=4 z^{2}-4 \alpha \pm 4+O\left(|z|^{-2}\right)$, which follows from (20), we obtain

$$
-Q(z)=\frac{w^{\prime}(z)^{2}}{2 w(z)^{2}}+\frac{3}{2} w(z)^{2}+2 z w(z)+2 z^{2}-2 \alpha=4 z^{2}-4 \alpha \pm 2+O\left(|z|^{-2}\right),
$$

hence essentially the same equation as in case of (iv) $)_{c}^{ \pm}$with $\gamma \neq 0$. The proof of uniqueness is much easier for equations (I) and (II); we obtain $u^{\prime \prime}+Q(z) u=0$ with $\tau=1 / 2$ and $\tau=1$, hence either $u \equiv 0$ or else $\Theta \leq \frac{4}{5} \pi$ and $\Theta \leq \frac{2}{3} \pi$, respectively.

6.3. Triply truncated first transcendents. Following Boutroux [4], Painlevé's equation (I) has five triply truncated solutions (also called tritronquée); for a recent existence proof see Joshi and Kitaev [20]. Since (I) is invariant under the transformation $w \mapsto a^{2} w(a z)$ with $a^{5}=1$, it suffices to prove the existence of a triply truncated solution $w_{0}$ having the asymptotics $w_{0}(z) \sim-\sqrt{-z / 6}$ with $\operatorname{Re} \sqrt{-z / 6}>0$ on $|\arg z-\pi|<4 \pi / 5$, which thus is truncated along $\sigma_{1}, \sigma_{2}$, and $\sigma_{3}$. By Theorem 6.1(i) there exist uniquely determined first transcendents $w_{1}$ and $w_{2}$ with asymptotics $w_{1,2}=-\sqrt{-z / 6}+O\left(|z|^{-2}\right)$ and $\operatorname{Re} \sqrt{-z / 6}>0$ on $\pi / 5<\arg z<7 \pi / 5$ and on $3 \pi / 5<\arg z<9 \pi / 5$, respectively. Then again $y=w_{1}-w_{2}$ satisfies

$$
y^{\prime \prime}=6\left(w_{1}(z)+w_{2}(z)\right) y=\left(-12 \sqrt{-z / 6}+O\left(|z|^{-2}\right)\right) y
$$

on $3 \pi / 5<\arg z<7 \pi / 5$. We set $z=-6 t^{4 / 5}$ and $y(z)=t^{-1 / 10} v(t)$ on the right half-plane $\operatorname{Re} t>0\left(t^{4 / 5}>0, \sqrt{-z / 6}=t^{2 / 5}>0\right.$ and $t^{-1 / 10}>0$ if $\left.t>0\right)$ to obtain

$$
100 \ddot{v}+\left[27648+9 t^{-2}+O\left(|t|^{-11 / 5}\right)\right] v=0 .
$$

Now every non-trivial solution to (28) tends to infinity exponentially as $t \rightarrow \infty$ at least on one of the sectors $\delta<\arg t<\pi / 2-\delta$ and $-\pi / 2+\delta<\arg t<-\delta$. This proves $v \equiv 0$ and $y \equiv 0$, and $w_{0}=w_{1}=w_{2}$ is truncated along $\sigma_{1}, \sigma_{2}$, and $\sigma_{3}$.

Remark 6.1. We note that $\overline{w_{0}(\bar{z})}$ is also a solution that is truncated along $\sigma_{1}, \sigma_{2}$, and $\sigma_{3}$, and hence coincides with $w_{0}$ by uniqueness. In other words, the tritronquée solution is real on the real axis. We also note that if $w$ is any solution that is truncated along $\sigma_{1}$ and $\sigma_{2}$ resp. $\sigma_{2}$ and $\sigma_{3}$ coincides with $w_{0}$ by Theorem 6.1 if it has the same asymptotics as $w_{0}$ on $\Sigma_{1} \cup \sigma_{1} \cup \Sigma_{2}$ resp. $\Sigma_{2} \cup \sigma_{2} \cup \Sigma_{3}$.

Remark 6.2. In contrast to Theorem 6.1 the triply truncated solution $w_{0}$ is not only unique but also determines its asymptotics. In other words, there is no solution $\tilde{w}_{0}$ that is truncated along $\sigma_{1}, \sigma_{2}$, and $\sigma_{3}$ and is asymptotic to $+\sqrt{-z / 6}$ with $\operatorname{Re} \sqrt{-z / 6}>0$. Otherwise we will consider the solution $v(z)=e^{4 \pi i / 5} \tilde{w}_{0}\left(e^{2 \pi i / 5} z\right)$, which is truncated along the Stokes rays $\sigma_{0}, \sigma_{1}$, and $\sigma_{2}$. Then $w_{0}, \tilde{w}_{0}$ and $v$ have asymptotic expansions on $\pi / 5<\arg z<7 \pi / 5$, which by Theorem 6.1 is only possible if two of these solutions agree, which obviously is not the case.

\section{The dichotomy of the order}

On combination with work of Mues and Redheffer [24] and Shimomura [30] it follows that first transcendents have order of growth $\frac{5}{2}$. The situation is quite different for second and fourth transcendents, they have order of growth between $\frac{3}{2}$ and 3 and between 2 and 4, respectively. More precisely they satisfy

$$
C_{1} r^{\frac{3}{2}} \leq T(r, w) \leq C_{2} r^{3} \quad \text { and } \quad C_{1} r^{2} \leq T(r, w) \leq C_{2} r^{4}
$$

in the respective cases, see $[16,17,30,38]$. 
7.1. Weber-Hermite solutions. The solutions to the Weber-Hermite equations

$$
w^{\prime}=2 \gamma \pm\left(2 z w+w^{2}\right) \quad(\gamma=-1 \mp \alpha)
$$

also solve equation (IV) with $\beta=-2(1 \pm \alpha)^{2}$, and satisfy $n(r, w) \asymp r^{2} .\left(^{4}\right)$ Repeated application of the Bäcklund transformations (22), (23), and the transformations $w(z) \mapsto-i w(i z)$ and $w \mapsto \overline{w(\bar{z})}$ to the solutions to (29) leads to the so-called Weber-Hermite solutions. Just like the solutions to (29) they have counting function of poles $n(r, w) \asymp r^{2}$. In section 8 it will be shown that the Weber-Hermite solutions coincide with those satisfying $n(r, w)=O\left(r^{2}\right)$. In case of (II) the role of the Weber-Hermite equations is taken by the Airy equations $w^{\prime}= \pm\left(z / 2+w^{2}\right)$ with $n(r, w) \asymp r^{3 / 2}$.

7.2. Solutions of maximal order of growth. It is not hard to identify the second and fourth transcendents of order 3 and 4 mean type, respectively.

Theorem 7.1. Let $w$ be any Painlevé transcendent whose cluster set contains some non-exceptional parameter $\mathfrak{c}_{0}$. Then $w$ has maximal order of growth. More precisely, there exist sequences $r_{k} \rightarrow \infty$ such that

$$
\text { (i) } n\left(r_{k}, w\right) \asymp r_{k}^{5 / 2}, \quad \text { (ii) } n\left(r_{k}, w\right) \asymp r_{k}^{3}, \quad \text { and } \text { (iv) } n\left(r_{k}, w\right) \asymp r_{k}^{4}
$$

holds in the respective case.

Remark 7.1. Hinkkanen and Laine [18] for equation (II), and Sriponpaew [34] and Classen [7] for equation (IV) provided different proofs of Theorem 7.1.

Proof. Again the idea is the same in all cases, as usual we prefer to consider fourth transcendents. There exists some sequence $\left(h_{k}\right)$ such that $h_{k}^{-3} W\left(h_{k}\right)$ tends to some constant $\mathfrak{c}_{0} \neq 0,-8 / 27$. Since $z^{-3} W(z)$ varies slowly, this following from the fact that

$$
\frac{d}{d z} z^{-3} W(z)=-3 z^{-4} W(z)+z^{-3}\left(2 z w(z)+w(z)^{2}\right)=O\left(|z|^{-1}\right)
$$

holds outside $\mathcal{P}_{\delta}$, there exists some $\epsilon>0$ such that

$$
\left|z^{-3} W(z)-\mathfrak{c}_{0}\right|<\frac{1}{2} \min \left\{\left|\mathfrak{c}_{0}\right|,\left|\mathfrak{c}_{0}+8 / 27\right|\right\}
$$

holds on $D_{k}=\left\{z:\left|z-h_{k}\right|<\epsilon\left|h_{k}\right|\right\} \backslash \mathcal{P}_{\delta} ; \delta>0$ is fixed. In other words, for every appropriate sequence $\left(\tilde{h}_{\nu}\right)$ with $\tilde{h}_{\nu} \in D_{k_{\nu}}$ the limit function $\mathfrak{w}=\lim _{\nu \rightarrow \infty} w_{\tilde{h}_{\nu}}$ is doubly periodic and solves some differential equation $\mathfrak{w}^{\prime 2}=\mathfrak{w}^{4}+4 \mathfrak{w}^{3}+4 \mathfrak{w}^{2}-4 \mathfrak{c} \mathfrak{w}$ with parameter satisfying $\left|\mathfrak{c}-\mathfrak{c}_{0}\right| \leq \frac{1}{2} \min \left\{\left|\mathfrak{c}_{0}\right|,\left|\mathfrak{c}_{0}+8 / 27\right|\right\}$, hence $|\mathfrak{c}| \geq \frac{1}{2}\left|\mathfrak{c}_{0}\right|$ and also $|\mathfrak{c}+8 / 27| \geq \frac{1}{2}\left|\mathfrak{c}_{0}+8 / 27\right|$. The corresponding lattice $\Lambda_{\mathfrak{c}}$ has a fundamental parallelogram whose diameter is bounded independent of $\mathfrak{c}$, hence there exist $R>0$ and $k_{0} \in \mathbf{N}$, such that for every $k \geq k_{0}$ and center $z_{0}$ satisfying $\left|z_{0}-h_{k}\right|<\epsilon\left|h_{k}\right|$, the disc $\mathrm{D}_{R}\left(z_{0}\right)=\left\{z:\left|z-z_{0}\right|<R\left|h_{k}\right|^{-1}\right\}$ contains at least one pole of $w$. Now the disc $\left|z-h_{k}\right|<\epsilon\left|h_{k}\right|$ contains $\asymp\left|h_{k}\right|^{4}$ centers $z_{\nu}$ of mutually disjoint discs $\mathrm{D}_{R}\left(z_{\nu}\right)$ (it is the same to say that, as $r \rightarrow \infty$, the disc $|\mathfrak{z}|<r$ contains $\asymp r^{2}$ integers $m+i n$ : just map $|\mathfrak{z}|<\frac{\epsilon}{2 R}\left|h_{k}\right|^{2}$ onto $\left|z-h_{k}\right|<\epsilon\left|h_{k}\right|$ by $\mathfrak{z} \mapsto h_{k}+2 R\left|h_{k}\right|^{-1} \mathfrak{z}$ ), and since the $\operatorname{discs} \mathrm{D}_{R}\left(z_{\nu}\right)$ are contained in $\left|z-h_{k}\right|<2\left|h_{k}\right|$, say, it follows that $n\left(2\left|h_{k}\right|, w\right) \geq \mu\left|h_{k}\right|^{4}$ holds for some $\mu>0$ that is independent of $k$. Together with $n(r, w)=O\left(r^{4}\right)$ this yields $n\left(r_{k}, w\right) \asymp r_{k}^{4}$ for the sequence $r_{k}=2\left|h_{k}\right| \rightarrow \infty$.

\footnotetext{
${ }^{4}$ More precisely, $n(r, w)=\nu(w) \frac{r^{2}}{2 \pi}+o\left(r^{2}\right)$ holds, were either $\nu(w)=4$ or else $\nu(w)=2[41]$.
} 
Remark 7.2. There is some evidence to believe that a much stronger result holds: Given any fourth [second] transcendent $w$ and any Stokes sector $\Sigma$, then either $w$ has an asymptotic expansion on $\Sigma$ or else the cluster set $\mathfrak{C}_{\Sigma^{*}}(w)$ of $w$ restricted to any closed sub-sector $\Sigma^{*}$ of $\Sigma$ contains neither 0 nor $-8 / 27$ [neither 0 nor $-1 / 4$ ]. In other words, either each or else none limit function $\mathfrak{w}=\lim _{h_{n} \rightarrow \infty} w_{h_{n}}$ with $\left(h_{n}\right) \subset \Sigma^{*}$ is constant.

\section{Sub-normal solutions}

Fourth transcendents satisfying $n(r, w)=O\left(r^{2}\right)$ as $r \rightarrow \infty$ are called sub-normal. We note that by Theorem 7.1, fourth transcendents satisfying $n(r, w)=o\left(r^{4}\right)$ have cluster set either $\mathfrak{C}(w)=\{0\}$ or else $\mathfrak{C}(w)=\{-8 / 27\}$. In particular, this is true for sub-normal solutions.

8.1. Sub-normal solutions with cluster set $\mathfrak{C}(\boldsymbol{w})=\{-8 / 27\}$. Similar to the case of equation (II), which was considered in [40], it turns out that the following is true.

Theorem 8.1. Sub-normal fourth Painlevé transcendents with cluster set $\mathfrak{C}(w)=$ $\{-8 / 27\}$ do not exist.

Proof. Let $w$ be any fourth transcendent with counting function of poles $n(r, w)=$ $O\left(r^{2}\right)$ as $r \rightarrow \infty$ and cluster set $\mathfrak{C}(w)=\{-8 / 27\}$. The re-scaling method then shows that every pole of sufficiently large modulus is contained in some uniquely determined string of poles $\mathfrak{p}=\left(p_{k}\right)$ satisfying

$$
p_{k+1}=p_{k} \pm(\pi \sqrt{3}+o(1)) p_{k}^{-1},
$$

hence $p_{k}$ is given by $p_{k}=( \pm(2 \pi \sqrt{3}+o(1)) k)^{\frac{1}{2}}$ with fixed sign \pm and fixed square-root (see Lemma 4.1). From our hypothesis and

$$
n(r, \mathfrak{p})=\frac{r^{2}}{2 \pi \sqrt{3}}+o\left(r^{2}\right)
$$

it follows that there are only finitely many such strings, each of them being asymptotic to some ray $\arg z=\nu \pi / 2$. Thus the corresponding Stokes sectors $\Sigma_{\nu}$ are 'polefree', and $w$ has one and the same asymptotic expansion (19)(iv) $)_{a}$ on each $\Sigma_{\nu}$. By Proposition 6.1 the asymptotic expansion (19)(iv) $)_{a}$ holds on the whole plane, hence $w$ is a rational function. This proves Theorem 8.1.

8.2. Sub-normal solutions with cluster set $\mathfrak{C}(\boldsymbol{w})=\{0\}$. We shall now prove the main theorem on sub-normal solutions. The corresponding result for second transcendents has been proved in [40]; Theorems 8.1 and 8.2 have also been proved by Classen [7].

Theorem 8.2. The sub-normal fourth transcendents with cluster set $\mathfrak{C}(w)=\{0\}$ coincide with the Weber-Hermite solutions.

Proof. Let $w$ be any sub-normal fourth transcendent with cluster set $\mathfrak{C}(w)=$ $\{0\}$. Again the re-scaling method, on combination with Lemma 4.1 with $\tau=1$ and $\omega= \pm \pi i$ shows that the set of poles of $w$ on $|z|>r_{0}$ consists of finitely many strings $\mathfrak{p}=\left(p_{k}\right)$, each being asymptotic to some ray $\arg z=(2 \nu+1) \pi / 4(0 \leq \nu \leq 3)$ and having counting function $n(r, \mathfrak{p})=r^{2} / 2 \pi+o\left(r^{2}\right)$. This implies

$$
n(r, w)=\nu(w) \frac{r^{2}}{2 \pi}+o\left(r^{2}\right)
$$


where $\nu(w)$ denotes the number of strings. On each sector $\Sigma_{\nu}:|\arg z-\nu \pi / 2|<$ $\pi / 4, w$ has an asymptotic expansion either $(19)(\mathrm{iv})_{b}$ or else (19)(iv) $)_{c}$. The Residue Theorem gives

$$
\begin{aligned}
& \frac{1}{2 \pi i} \int_{\Gamma_{r}} w(z) d z=\Delta(w) \frac{r^{2}}{2 \pi}+o\left(r^{2}\right) \quad \text { and } \\
& \frac{1}{2 \pi i} \int_{\Gamma_{r}} W(z) d z=-n(r, w) \quad\left(\text { note that } \operatorname{res}_{p} W=-1\right),
\end{aligned}
$$

where $\Gamma_{r}$ denotes the loop that was constructed in Section 2.3, and $\Delta(w)$ denotes the difference between the number of strings $\mathfrak{p}$ with residue +1 and -1 . Since $|w(z)|=$ $O(|z|)$ holds on $\Gamma_{r}$, and the length of the part of $\Gamma_{r}$ that is contained in $\mid \arg z-(2 \nu+$ 1) $\pi / 4 \mid<\epsilon$ is at most $4 \pi \epsilon r$, the contribution of the sector $\Sigma_{\nu}$ to the first integral (31) is $o\left(r^{2}\right)$ if $w$ has the asymptotic expansion $w \sim \pm \gamma / z+\cdots$, and is $(-1)^{\nu-1} r^{2} / \pi+o\left(r^{2}\right)$ if $w \sim-2 z+\cdots$. In other words, the contribution of the sector $\Sigma_{\nu}$ to $\Delta(w)$ is 0 if $w \sim \pm \gamma / z+\cdots$, and is $-2(-1)^{\nu}$ if $w \sim-2 z+\cdots$ holds on $\Sigma_{\nu}$. We apply the Bäcklund transformation (22) to obtain

$$
w_{1}=\frac{w^{\prime}-2 \gamma-2 z w-w^{2}}{2 w}
$$

and conclude from (25) and (31) that

$$
n\left(r, w_{1}\right)-n(r, w)=\left(\Delta(w)+\Delta\left(w_{1}\right)\right) \frac{r^{2}}{2 \pi}+o\left(r^{2}\right) .
$$

The main idea of proof now is to determine some appropriate Bäcklund transformation such that $\Delta(w)+\Delta\left(w_{1}\right)$ is negative. To this end we introduce the signature $\left[a_{2} a_{1} a_{3} a_{0}\right]$ of $w$ to indicate that

$$
W=2 a_{\nu} z+\frac{\lambda_{\nu}}{z}+O\left(|z|^{-3}\right)
$$

holds on the Stokes sector $\Sigma_{\nu}$, uniformly on every closed sub-sector, with $a_{\nu} \in$ $\{-\gamma, \gamma, \alpha\}$ and $\lambda_{\nu}$ according to (21). Before going into details we shall study the entire function $F(z)=e^{-\int W(z) d z}$ with corresponding signature $\left[a_{2} a_{1} a_{3} a_{0}\right]$ of $w$. Corollary 11.1 in Appendix A, applied to

$$
h(\zeta)=F\left(e^{\frac{1}{4}(2 \nu+1) \pi i} \sqrt{\zeta}\right) \quad(\sqrt{\zeta}>0 \text { if } \zeta>0)
$$

with $a=(-1)^{\nu-1} i a_{\nu+1}$ and $b=(-1)^{\nu-1} i a_{\nu}$ then yields $\operatorname{Re} a=\operatorname{Re} b$ and $\operatorname{Im} a \leq \operatorname{Im} b$, hence

$$
\operatorname{Im} a_{\nu+1}=\operatorname{Im} a_{\nu} \quad \text { and } \quad(-1)^{\nu-1} \operatorname{Re} a_{\nu+1} \leq(-1)^{\nu-1} \operatorname{Re} a_{\nu}
$$

in other words, the differences $a_{1}-a_{0}, a_{1}-a_{2}, a_{3}-a_{2}$ and $a_{3}-a_{0}$ are real and nonnegative. Moreover, $a_{\nu+1}=a_{\nu}$ implies $\lambda_{\nu+1}=\lambda_{\nu}$, and again from the PhragménLindelöf Principle it follows that the half-plane $\mathrm{H}_{\nu}:|\arg z-(2 \nu+1) \pi / 4|<\pi / 2$ is 'pole-free' and thus $w$ has an asymptotic expansion on $\mathrm{H}_{\nu}$ by Proposition 6.1.

We are now looking for an appropriate starting point for the final argument.

a. The change of variables $v(z)=\overline{w(\bar{z})}$ transforms (IV) into equation (IV) for $v$ with parameters $\bar{\alpha}$ and $\bar{\beta}$ and first integral $V(z)=\overline{W(\bar{z})}$, while the signature $\left[\begin{array}{cc}a_{2} & a_{1} \\ a_{3} & a_{0}\end{array}\right]$ changes into $\left[\begin{array}{c}\bar{a}_{2} \\ \bar{a}_{3} \\ \bar{a}_{1}\end{array} \bar{a}_{0}\right]$. We may thus formally interchange the positions of $a_{1}$ and $a_{3}$, while the positions of $a_{0}$ and $a_{2}$ remain fixed. 
b. The change of variables $v(z)=-i w(i z)$ transforms (IV) into equation (IV) for $v$ with parameters $-\alpha$ and $\beta$, while the asymptotic expansions $W \sim 2 \alpha z$ and $W \sim \pm 2 \gamma z$ are changed into $V \sim-2 \alpha z$ and $V \sim \mp 2 \gamma z$, respectively.

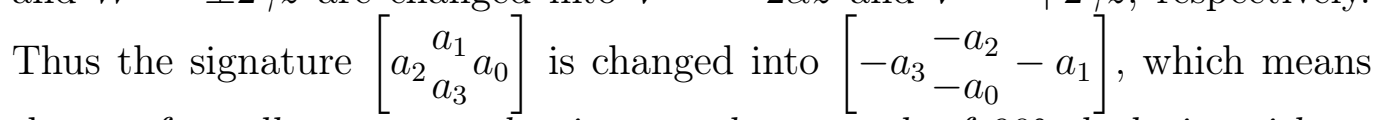
that we formally may turn the signature by an angle of $90^{\circ}$ clockwise without changing our hypotheses.

Applying these transformations (which, in an abstract sense, generate the dihedral group $D_{4}$ acting on signatures) several times, if necessary, it is easily seen that there are five abstract combinatorial configurations to be discussed:
1. $\left[b_{b}^{a} a\right]$,
2. $[a b b]$
3. $\left[a_{b}^{a} a\right]$,
4. $\left[a_{c}^{b} a\right]$, and
5. $\left[c^{a} b\right]$

with $a, b, c \in\{\alpha,-\gamma, \gamma\}$; although $a$ and $b$, say, may be numerically equal, they represent different symbols. For example, applying first b. followed by a. yields $\left[\begin{array}{c}c \\ a \\ a\end{array}\right] \mapsto\left[-a^{-c}-a-a\right] \mapsto[-\bar{a}-\bar{c}-\bar{a}]$; the latter formally has the shape of the above signature 4. By applying Proposition 5.1, if necessary, we may identify $a$ with $\alpha$, and since we are free to choose the branch of $\gamma=\sqrt{-\beta / 2}$, in other words to replace $\gamma$ by $-\gamma$ in (22), we may assume $b=-\gamma$ and $c=\gamma$, this leading to five concrete configurations as follows:
1. $\left[-{ }_{-\gamma}^{\alpha} \alpha\right]$,
2. $\left[{ }^{-\gamma}-\gamma \alpha\right]$
3. $\left[{ }_{-\gamma}^{\alpha} \alpha\right]$
4. $\left[\begin{array}{c}\alpha-\gamma \\ \gamma\end{array}\right]$,
5. $\left[\gamma_{-\gamma}^{\alpha} \alpha\right]$

In the first case, $w$ has the asymptotic expansions (19)(iv) $)_{b}$ on the right upper half-plane $\operatorname{Im} z>-\operatorname{Re} z$, and (19)(iv) ${ }_{c}^{-}$on $\operatorname{Im} z<-\operatorname{Re} z$. Thus (33) applies with $\nu=1: a_{1}=\alpha, a_{2}=-\gamma$, and $\nu=3: a_{3}=-\gamma, a_{4}=a_{0}=\alpha$, this yielding $\alpha=-\gamma$. From Corollary 11.1 in Appendix A it then follows that $w$ has only finitely many poles, hence is a rational function in contrast to our assumption.

We will now show that none of the remaining cases 2 . to 5 . can occur for nonWeber-Hermite sub-normal solutions. Non-Weber-Hermite solutions admit unrestricted application of the Bäcklund transformation (22), this leading to sequences $\left(\alpha_{k}\right)$ and $\left(\gamma_{k}\right)$ of parameters: $\alpha, \alpha_{1}, \alpha+1, \alpha_{1}+1, \ldots$ and $\gamma, \gamma_{1}, \gamma+1, \gamma_{1}+1, \ldots$ We have to distinguish two cases as follows:

Case a. $\gamma_{k} \neq 0$ as long as $w_{k} \not \equiv 0$. Applying (22) twice we obtain in case 2.

$$
\left[\begin{array}{c}
\alpha^{-\gamma}{ }_{-\gamma} \\
\alpha
\end{array}\right] \mapsto\left[{ }^{-}{ }^{-\gamma_{1}} \gamma_{1}\right] \mapsto\left[\begin{array}{c}
-\gamma_{2} \\
\alpha_{2}-\gamma_{2}
\end{array} \alpha_{2}\right], \quad \Delta(w)+\Delta\left(w_{1}\right)=-4+0=-4
$$

From (32) (it is obvious that we may construct $\Gamma_{r}$ in such a way that it simultaneously works for $w$ and $\left.w_{1}\right)$ it follows that

$$
n\left(r, w_{1}\right)-n(r, w)=-4 \frac{r^{2}}{2 \pi}+o\left(r^{2}\right)
$$

Repeating this process we obtain by 2-periodicity of the sequence $\left(\Delta\left(w_{k}\right)\right)$ and the corresponding sequence of signatures

$$
n\left(r, w_{k}\right)=n(r, w)-4 k \frac{r^{2}}{2 \pi}+o\left(r^{2}\right)
$$

in other words, at every step four strings of poles get lost. This, however, cannot be true for every $k \in \mathbf{N}$, that is, starting with some sub-normal solution $w$ with 
signature 2., there exists some $k$ such that

$$
w_{k+1}=\frac{w_{k}^{\prime}-2 \gamma_{k}-2 z w_{k}-w_{k}^{2}}{2 w_{k}} \equiv 0,
$$

and $w_{k}$ satisfies the Weber-Hermite equation $w_{k}^{\prime}=2 \gamma_{k}+2 z w_{k}+w_{k}^{2}$ and has signature $\left[\alpha_{k}-\gamma_{k} \alpha_{k}\right]$. The argument is similar in all other cases:

$$
\begin{aligned}
& \text { 3. }\left[\begin{array}{c}
\alpha \\
\alpha-\gamma
\end{array} \alpha\right] \mapsto\left[\gamma_{1}{ }_{-\gamma}^{\gamma_{1}} \gamma_{1}\right] \mapsto\left[\begin{array}{c}
\alpha_{2} \\
\alpha_{2}-\gamma_{2}
\end{array} \alpha_{2}\right], \quad \Delta(w)+\Delta\left(w_{1}\right)=-2+0=-2 \text {, } \\
& \text { 4. }\left[\begin{array}{c}
\alpha_{\gamma}^{-\gamma} \alpha \\
\gamma
\end{array}\right] \mapsto\left[\begin{array}{c}
-\gamma_{1} \\
\gamma_{1} \alpha_{1}
\end{array} \alpha_{1}\right] \mapsto\left[\begin{array}{c}
-\gamma_{2} \\
\alpha_{2} \alpha_{2}
\end{array}\right], \quad \Delta(w)+\Delta\left(w_{1}\right)=-4+2=-2 \text {, } \\
& \text { 5. }\left[\begin{array}{c}
\alpha \\
\gamma_{-\gamma}^{\alpha}
\end{array}\right] \mapsto\left[\begin{array}{c}
\gamma_{1} \\
\alpha_{1} \gamma_{1}
\end{array}\right] \mapsto\left[\begin{array}{c}
\alpha_{2} \\
\gamma_{2}-\gamma_{2}
\end{array} \alpha_{2}\right], \quad \Delta(w)+\Delta\left(w_{1}\right)=0-2=-2 .
\end{aligned}
$$

Thus $n\left(r, w_{k}\right)=n(r, w)-k r^{2} / \pi+o\left(r^{2}\right)$ holds in all cases, this leading to the same conclusion as in case $\mathbf{2}$. A posteriori it turns out that the cases $\mathbf{4}$. and $\mathbf{5}$. will never occur $(\gamma \neq 0)$.

Case b. $\gamma=0$. Here we just have to consider the cases 2. and 3., where the first step leads to $w_{1}$ with signature $\left[\gamma_{1}-\gamma_{1} \gamma_{1}\right]$ and $\left[\begin{array}{c}\gamma_{1} \\ \gamma_{1}-\gamma_{1}\end{array} \gamma_{1}\right]$, respectively. Then $\gamma_{1}=0$ implies $w_{1} \equiv 0$ and we are done, while otherwise we may proceed like in Case a. This completes the proof of Theorem 8.2.

8.3. Rational and sub-normal solutions. Rational solutions to equation $(\mathrm{IV})_{\alpha, \beta}$ are uniquely determined. They occur in three shapes $w=-2 z+\cdots, w=$ $\gamma / z+\cdots\left(\gamma^{2}=-\beta / 2\right)$, and $w=-\frac{2}{3} z+\cdots$ for certain well-known parameters, see [11], $\S 26$. The sub-normal (= Weber-Hermite) solutions to $(\mathrm{IV})_{\alpha, \beta}$ form a one-parameter family and occur if and only if either $\beta=-2(1+2 n-\alpha)^{2}$ or else $\beta=-2 n^{2}$, while $\alpha$ is arbitrary. For $\alpha \notin \mathbf{Z}$ there exist four sub-normal solutions that are truncated along two adjacent rays, while for $\alpha \in \mathbf{Z}$ their role is taken by rational solutions. Any other solution satisfies $r^{2}=o(n(r, w))$.

8.4. Sub-normal second transcendents. The analogs to Theorem 8.1 and 8.2 have been proved in [40]. We will now show how the proof of Theorem 8.1 and 8.2 may be adapted to this case to obtain a proof that is quite different from the original proof in [40].

Sketch of proof. Let $w$ be any sub-normal solution $\left(n(r, w)=O\left(r^{3 / 2}\right)\right)$ to equation (II). Then $w$ has cluster set either $\mathfrak{C}(w)=\{-1 / 4\}$ or else $\mathfrak{C}(w)=\{0\}$. The poles of $w$ are arranged in finitely many strings; each string has counting function $n(r, \mathfrak{p})=$ $\frac{\sqrt{2}}{3 \pi} r^{3 / 2}+o\left(r^{3 / 2}\right)$ and $n(r, \mathfrak{p})=\frac{1}{3 \pi} r^{3 / 2}+o\left(r^{3 / 2}\right)$, and is asymptotic to some Stokes ray $\arg z=2 \nu \pi / 3$ and $\arg z=(2 \nu+1) \pi / 3$, respectively. On the sectors between the Stokes rays, $w$ has asymptotic expansions $(19)(\mathrm{ii})_{b}$ and (ii) $)_{a}$, respectively. The cluster set, the asymptotics, and the growth of the Nevanlinna characteristic are invariant under the Bäcklund transformations

$$
\begin{aligned}
& B_{+}: w \mapsto \tilde{w}=-w-\frac{\alpha+1 / 2}{w^{\prime}+w^{2}+z / 2} \quad(\alpha \neq-1 / 2, \alpha \mapsto \alpha+1=\tilde{\alpha}), \\
& B_{-}: \tilde{w} \mapsto w=-\tilde{w}+\frac{\tilde{\alpha}-1 / 2}{\tilde{w}^{\prime}-\tilde{w}^{2}-z / 2} \quad(\tilde{\alpha} \neq 1 / 2, \tilde{\alpha} \mapsto \tilde{\alpha}-1=\alpha) .
\end{aligned}
$$


Set $\tilde{w}=B_{+}[w]$ and observe that $w+\tilde{w}=\frac{\alpha+1 / 2}{\tilde{w}^{\prime}-\tilde{w}^{2}-z / 2}=-\frac{\alpha+1 / 2}{w^{\prime}+w^{2}+z / 2}$, hence

$$
w^{\prime}+\tilde{w}^{\prime}=-w^{2}+\tilde{w}^{2}=-W^{\prime}+\tilde{W}^{\prime} \text { and } \quad \tilde{W}-W=\tilde{w}+w+\text { const. }
$$

This implies $\tilde{W}-W=O\left(|z|^{1 / 2}\right)\left(z \notin \mathcal{P}_{\delta}(w) \cup \mathcal{P}_{\delta}(\tilde{w})\right)$ and $\mathfrak{C}(\tilde{w})=\mathfrak{C}(w)$. Subnormal solutions with $\mathfrak{C}(w)=\{0\}$ do not exist; the proof is the same as proof of Theorem 8.1. For $\mathfrak{C}(w)=\{-1 / 4\}$, the number $\Delta(w)$, which denotes the difference between the number of strings with residue 1 and -1 , respectively, is non-zero and invariant under (34); actually $\Delta(w)= \pm 1$ or else $\Delta(w)= \pm 3$, since the contribution to $\Delta(w)$ of each sector $2 \nu \pi / 3<\arg z<(2 \nu+2) \pi / 3$ is 1 or -1 . Replacing $w$ by $-w$, if necessary, one may assume $\Delta(w)>0$, and with $\tilde{w}=B_{+}[w]$ it follows that

$$
\begin{aligned}
n(r, w)-n(r, \tilde{w}) & =\frac{1}{2 \pi i} \int_{\Gamma_{r}}(\tilde{W}(z)-W(z)) d z \\
& =\frac{1}{2 \pi i} \int_{\Gamma_{r}}(\tilde{w}(z)+w(z)) d z=2 \Delta(w) \frac{\sqrt{2}}{3 \pi} r^{3 / 2}+o\left(r^{3 / 2}\right) .
\end{aligned}
$$

Remark 8.1. The Airy equation $w^{\prime}=z / 2+w^{2}$ has three uniquely determined solutions with $\Delta(w)=-1$ and a single string of poles, see [41]; $k$-fold application of the above Bäcklund transformation yields Airy solutions with $2 k+1$ strings in the same direction; $k+1$ strings have $\operatorname{res}_{\mathfrak{p}} w=-1$ and $k$ strings have $\operatorname{res}_{\mathfrak{p}} w=+1$, thus $n\left(r, w_{k}\right) \sim(2 k+1) \sqrt{2} r^{3 / 2} / 3 \pi$. In the generic case there are three 'active' directions, $\Delta(w)=-3$ and $n\left(r, w_{k}\right) \sim(2 k+1) \sqrt{2} r^{3 / 2} / \pi$.

\section{The distribution of zeros and poles}

9.1. Equivalence classes and the order of sub-normal solutions. Fourth transcendents $w_{1}$ and $w_{2}$ are called equivalent, if $w_{1}$ and $w_{2}$ are linked by some trivial Bäcklund transformation (any combination of rotations $w_{2}(z)=\eta w_{1}(\bar{\eta} z)$ with $\eta^{4}=1$ and reflections $\left.w_{2}(z)=\overline{w_{1}(\bar{z})}\right)$. Sub-normal transcendents $w$ are mapped by repeated application of Bäcklund transformations onto some solution of one of the Weber-Hermite equations (29): $w_{0}^{\prime}=2 \gamma_{0} \pm\left(2 z w_{0}+w_{0}^{2}\right), \gamma_{0}=-1 \mp \alpha_{0}$. The smallest number of non-trivial transformations needed is called the order of $w$. Subnormal solutions have strings of poles either in each Stokes direction (generic case) or else only in two consecutive directions (exceptional case); exceptional solutions are truncated along two Stokes rays; they are uniquely determined and exist if and only if the final equation (29) has no rational solution. Henceforth we will restrict to the generic case, and leave the exceptional case to the interested reader. From (24) and the proof of Theorem 8.2 we obtain:

Theorem 9.1. The equivalence classes of generic sub-normal solutions of even and odd order $2 k$ and $2 k-1$ are represented by sub-normal solutions with signature

$$
\left[\alpha^{-\gamma} \alpha\right](\alpha+\gamma=-2 k-1)
$$

and

$$
\left[\gamma_{-\gamma}^{-\gamma} \gamma\right](\gamma=-k)
$$

respectively; $\alpha$ is arbitrary and $\beta=-2 \gamma^{2}$. In each Stokes direction these solutions have $k+1$ strings of poles with residue -1 and $k$ strings of poles with residue 1 if the order is even, and $k$ strings of poles each with residue \pm 1 if the order is odd. 
Remark 9.1. Any solution of order $2 k$ and $2 k-1$ with signatures (35) and (36), respectively, is embedded in a chain

$$
\begin{aligned}
& \ldots \stackrel{(22)}{\rightarrow} w_{2 k} \stackrel{(22)}{\rightarrow} w_{2 k-1} \stackrel{(22)}{\rightarrow} \cdots \stackrel{(22)}{\rightarrow} w_{1} \stackrel{(22)}{\rightarrow} w_{0} \\
& w_{0}^{\prime}=2 \gamma_{0}+2 z w_{0}+w_{0}^{2} \quad\left(\alpha_{0}+\gamma_{0}=-1, \operatorname{res}_{p} w_{0}=1\right) \text {. }
\end{aligned}
$$

We note that the case $\gamma_{0}=0$ does not occur, since otherwise

$$
w_{1}=-\frac{w_{0}^{\prime}+2 z w_{0}+w_{0}^{2}}{2 w_{0}}=-2 z-w_{0}
$$

has order 1 and solves $w_{1}^{\prime}=-2+2 z w_{1}+w_{1}^{2}\left(\gamma_{1}=-1, \alpha_{1}=0\right)$, in contrast to the definition of the order.

9.2. The distribution of residues. Let $w$ be any sub-normal fourth transcendent. With each string of poles $\mathfrak{p}=\left(p_{k}\right)$ we will associate a polygon $\pi(\mathfrak{p})$ with vertices $p_{k}$. These polygons divide $|z|>R$ (sufficiently large) into domains $D_{\nu}$, arranged in cyclic order; $\pi_{\nu}=\pi\left(\mathfrak{p}_{\nu}\right)$ separates $D_{\nu}$ from $D_{\nu-1}$. Also $D_{\nu} \cap\{z:|z|=r\}$ $(r>R)$ is an arc of angular measure $\Theta_{\nu}(r)$, where $\Theta_{\nu}(r)$ either tends to $\frac{3}{4} \pi, \frac{\pi}{4}$ or else 0 as $r \rightarrow \infty$; in any case $r \Theta_{\nu}(r)$ tends to infinity. The polygon $\pi_{\nu}$ is accompanied by polygons $\hat{\pi}_{\nu-1}$ and $\tilde{\pi}_{\nu}$ in $D_{\nu-1}$ and $D_{\nu}$, respectively, which start at $|z|=R$ such that $\arg z \rightarrow k \frac{\pi}{4}$ with $k=k_{\nu}$ as $|z| \rightarrow \infty$ on $\hat{\pi}_{\nu-1}$ and $\tilde{\pi}_{\nu}$, and the angular measure and the length of the shorter arc on $|z|=r$ joining these polygons to $\pi_{\nu}$ tends to zero and infinity, respectively, as $r \rightarrow \infty$. Re-scaling along any sequence $\left(h_{n}\right)$ on $D_{\nu}$ with $\left|h_{n}\right| \operatorname{dist}\left(h_{n}, \partial D_{\nu}\right) \rightarrow \infty$ yields a constant limit function $\mathfrak{w} \equiv \tau_{\nu} \in\{0,-2\}$; in particular, $w=\tau_{\nu} z+o(|z|)$ holds as $z \rightarrow \infty$ on $\tilde{\pi}_{\nu}$, and similarly we obtain $w=\tau_{\nu-1} z+o(|z|)$ on $\hat{\pi}_{\nu-1}$. To determine $\epsilon_{\nu}=\operatorname{res}_{\mathfrak{p}_{\nu}} w \in\{-1,1\}$ we assume for simplicity $\arg z \sim \pi / 4$ on $\pi_{\nu}$, and compute

$$
\frac{1}{2 \pi i} \int_{\kappa_{\nu}(r)} w(z) d z=\epsilon_{\nu} \frac{r^{2}}{2 \pi}+o\left(r^{2}\right)
$$

along the positively oriented simple closed curve $\kappa_{\nu}(r)$ which consists of sub-arcs $\hat{\kappa}_{\nu-1}(r)$ and $\tilde{\kappa}_{\nu}(r)$ of $\hat{\pi}_{\nu-1}$ and $\tilde{\pi}_{\nu}$ joining $|z|=r_{0}$ to $|z|=r$, respectively, and sub$\operatorname{arcs} \sigma_{r_{0}}$ of $|z|=r_{0}>R$ and $\sigma_{r}$ of $|z|=r>r_{0}$. The latter has length $o(r)$, hence $\frac{1}{2 \pi i} \int_{\sigma_{r}} w(z) d z=o\left(r^{2}\right)$ holds. From $w(z)=\tau_{\nu-1} z+o(|z|)$ and $w(z)=\tau_{\nu} z+o(|z|)$ on $\hat{\pi}_{\nu-1}$ and $\tilde{\pi}_{\nu}$, respectively, and $\arg z \rightarrow \pi / 4$ as $|z| \rightarrow \infty$ we obtain

$$
\frac{1}{2 \pi i} \int_{\kappa_{\nu}(r)} w(z) d z=\frac{\left(\tau_{\nu-1}-\tau_{\nu}\right) r^{2}}{4 \pi}+o\left(r^{2}\right),
$$

which implies $\epsilon_{\nu}=\left(\tau_{\nu-1}-\tau_{\nu}\right) / 2$. The relation between the asymptotics and the residues in different Stokes directions is displayed in Table 1.

\begin{tabular}{|c|r|r|r|}
\hline Stokes ray & $\tau_{\nu-1}$ & \multicolumn{1}{|c|}{$\tau_{\nu}$} & \multicolumn{1}{|c|}{$\epsilon_{\nu}$} \\
\hline $\arg z=\frac{\pi}{4}, \frac{5}{4} \pi$ & 0 & -2 & 1 \\
& -2 & 0 & -1 \\
\hline $\arg z=\frac{3}{4} \pi, \frac{7}{4} \pi$ & 0 & -2 & -1 \\
& -2 & 0 & 1 \\
\hline
\end{tabular}

Table 1. Asymptotics and distribution of residues.

Example 9.1. Any sub-normal solution $w$ with signature $\left[\begin{array}{c}\alpha_{-\gamma}^{-\gamma} \alpha \\ \alpha\end{array}\right]$ (and parameter $\gamma=-(2 k+1+\alpha))$ has $2 k+1$ strings of poles asymptotic to $\arg z=\pi / 4$; in counter-clockwise order the residues are $(-1,+1,-1, \ldots,+1,-1)$. If $w$ has signature 
$\left[k^{-k}-k, k\right.$, the number of strings is $2 k$, and the residue vector in the same direction is given by $(+1,-1, \ldots,+1,-1)$, see also Table 2 below.

9.3. The distribution of zeros. The re-scaling method does not immediately apply to detect the zeros of $w$, since re-scaling of any fourth transcendent along any sequence of zeros yields the limit function $\mathfrak{w} \equiv 0$. The reason for this is that $w^{\prime}= \pm 2 \gamma$ at zeros is 'small', hence the initial values for the limit function are $\mathfrak{w}(0)=\mathfrak{w}^{\prime}(0)=0$. Nevertheless it is possible to determine the distribution of zeros in any case, and, in particular, for sub-normal solutions. The zeros of $w$ with $w^{\prime}= \pm 2 \gamma$ are poles of $w_{ \pm}=\frac{w^{\prime} \pm 2 \gamma}{2 w}+z+\frac{w}{2}$ with residue 1 . We thus may conclude that the zeros are distributed in the same manner as are the poles. In particular, they form strings if $w$ is sub-normal.

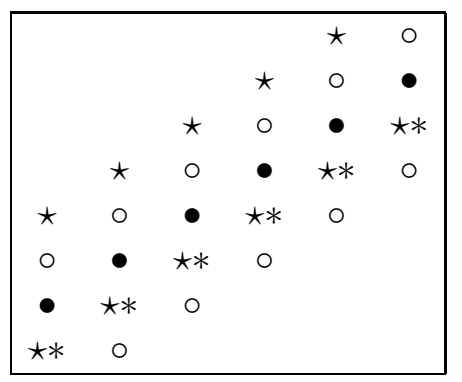

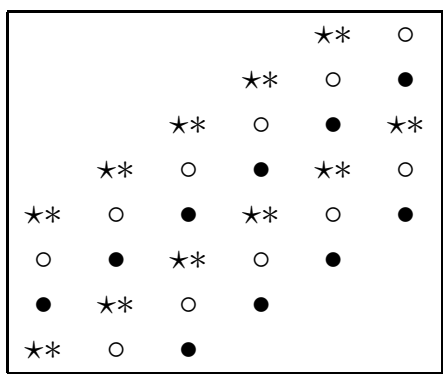

Table 2. Distribution of poles $\bullet \circ($ res $\bullet=1$, res。 $w=-1)$ and zeros $\star *$ along $\arg z=\pi / 4$; $w$ has signature (35) and (36), and order 2 and 3, respectively. In case of $\gamma=0$ the double string of zeros $\star *$ on the left hand side collapses to a string of double zeros, while the single string $\star$ disappears. The deficiency of zero then is $\delta(0, w)=1 / 3$.

9.4. Painlevé transcendents and first order differential equations. It is obvious that every Airy- and Weber-Hermite solution also satisfies some first order algebraic differential equation

$$
P\left(z, w, w^{\prime}\right)=w^{\prime n}+\sum_{\nu=1}^{n-1} P_{\nu}(z, w) w^{\prime \nu}=0 ;
$$

$P_{\nu}$ is a polynomial in $w$ (of degree $\leq 2 n-2 \nu$ ) over the field of rational functions in $z$. The converse was proved in [11], Theorem 21.1 and Theorem 25.4 for second and fourth transcendents, respectively. Based on the re-scaling method we will give a quite different proof of

Theorem 9.2. The second and fourth Painlevé transcendents also satisfying some first order algebraic differential equation (38) coincide with the Airy- and Weber-Hermite solutions, respectively, while first Painlevé transcendents never solve first order equations.

Proof. We consider solutions to (IV) which also solve (38) and assume that

$$
P(z, x, y)=\prod_{\nu=1}^{n}\left(y-G_{\nu}(z, x)\right)
$$

is irreducible. From $P\left(h+h^{-1} \mathfrak{z}, h w_{h}(\mathfrak{z}), h^{2} w_{h}^{\prime}(\mathfrak{z})\right)=0$ it then follows that the limit functions of the re-scaling process satisfy some Briot-Bouquet differential equation

$$
Q\left(\mathfrak{w}, \mathfrak{w}^{\prime}\right)=0
$$


with $Q(\mathfrak{x}, \mathfrak{y})=\lim _{h \rightarrow \infty} h^{-2 n} P\left(h, h \mathfrak{x}, h^{2} \mathfrak{y}\right)=\lim _{h \rightarrow \infty} h^{-2 n} \prod_{\nu=1}^{n}\left(h^{2} \mathfrak{y}-G_{\nu}(h, h \mathfrak{x})\right)$. On the other hand it is known that at any pole $p \neq 0$ with residue $\epsilon$,

$$
\epsilon w^{\prime}+2 z w+w^{2}=O\left(|p|^{2}\right) \quad(p \rightarrow \infty)
$$

holds on $|z-p|<\delta|p|^{-1}$. This implies $G_{\nu}(z, x)=\epsilon_{\nu} x^{2}+2 \epsilon_{\nu} z x+O\left(|z|^{2}\right)$ as $x \rightarrow \infty$, uniformly with respect to $z,|z-p|<\delta|p|^{-1}$. Re-scaling about any sequence of poles yields

$$
Q\left(\mathfrak{w}, \mathfrak{w}^{\prime}\right)=\prod_{\nu=1}^{n}\left(\mathfrak{w}^{\prime}-\epsilon_{\nu}\left(\mathfrak{w}^{2}+2 \mathfrak{w}+\mathfrak{a}_{\nu}\right)\right)=0 \quad\left(\epsilon_{\nu}= \pm 1, \mathfrak{a}_{\nu} \in \mathbf{C}\right) .
$$

Thus $\mathfrak{w}$ satisfies $\mathfrak{w}^{\prime}=\epsilon\left(\mathfrak{a}+2 \mathfrak{w}+\mathfrak{w}^{2}\right)$ for some $\epsilon \in\{1,-1\}$ and $\mathfrak{a} \in \mathbf{C}$, which is compatible with $(11)$ (iv) if and only if $\mathfrak{a}=\mathfrak{c}=0$. In particular, it follows that $w$ has cluster set $\mathfrak{C}(w)=\{0\}$, hence the set $\mathcal{P}$ of poles has string structure, that is, $\mathcal{P}$ consists of finitely or infinitely many strings of poles. In the first case $w$ is subnormal, hence a Weber-Hermite solution. To rule out the second possibility we have to discuss two subcases, which occur at every first order differential equation (see Eremenko $[8,9]$ ): equation (38) has genus either $g=0$ or else $g=1$. In the first case we have $w=R(z, y)$, where $R$ is rational and $y$ satisfies some Riccati equation with rational coefficients. Solutions to Riccati equations have only finitely many strings of poles, and this also holds for $w$ itself. In the second case, $w$ is a rational function of $u$ and $u^{\prime}$ over the field of algebraic functions, where $u$ satisfies some differential equation

$$
u^{\prime 2}=4 a(z)\left(w-e_{1}\right)\left(w-e_{2}\right)\left(w-e_{3}\right)
$$

with $e_{\mu} \neq e_{\nu}$ for $\mu \neq \nu, e_{1}+e_{2}+e_{3}=0$, and $a$ an algebraic function (thus $u(z)=$ $\wp\left(\int \sqrt{a(z)} d z\right)$ locally). This also leads to a contradiction, since this time the set $\mathcal{P}$ of poles locally has lattice structure in contrast to its string structure.

The proof for second transcendents runs along the same way. It remains to prove that first transcendents do not solve any first order differential equation (Theorem 13.1 in [11]). This time we obtain the Briot-Bouquet differential equation

$$
Q\left(\mathfrak{w}, \mathfrak{w}^{\prime}\right)=\prod_{\nu=1}^{n}\left(\mathfrak{w}^{\prime}-2 \epsilon_{\nu} \mathfrak{w}^{3 / 2}-\mathfrak{a}_{\nu}\right)=0 \quad\left(\epsilon_{\nu}= \pm 1, \mathfrak{a}_{\nu} \in \mathbf{C}\right),
$$

in contrast to $\mathfrak{w}^{\prime 2}=4 \mathfrak{w}^{3}+2 \mathfrak{w}-2 \mathfrak{c}$.

Example 9.2. To obtain first order equations for sub-normal solutions start with $w_{0}^{\prime}=2 \gamma_{0}+2 z w_{0}+w_{0}^{2}\left(\alpha_{0}=-1-\gamma_{0}\right)$, say, and compute successively (and in a purely algebraic manner)

$$
w_{\nu+1}=-\frac{w_{\nu}^{\prime}+2 \gamma_{\nu}+2 z w_{\nu}+w_{\nu}^{2}}{2 w_{\nu}}=\frac{P_{\nu}\left(z, w_{0}\right)}{Q_{\nu}\left(z, w_{0}\right)} \quad \text { and } \quad w_{\nu+1}^{\prime}=\frac{\tilde{P}_{\nu}\left(z, w_{0}\right)}{\tilde{Q}_{\nu}\left(z, w_{0}\right)} .
$$

The resultant with respect to $w_{0}$ of the polynomials $w_{\nu+1} Q_{\nu}\left(z, w_{0}\right)-P_{\nu}\left(z, w_{0}\right)$ and $w_{\nu+1}^{\prime} \tilde{Q}_{\nu}\left(z, w_{0}\right)-\tilde{P}_{\nu}\left(z, w_{0}\right)$ is a polynomial $R_{\nu+1}\left(z, w_{\nu+1}, w_{\nu+1}^{\prime}\right)$. Then $w=w_{k}$ is sub-normal of order $k$ and satisfies $R_{k}\left(z, w, w^{\prime}\right)=0$; the parameter $\alpha=\alpha_{k}$ may be prescribed by adjusting $\gamma_{0}$, while $\gamma=\gamma_{k}$ then is fixed. In the first step we obtain

$$
w^{\prime 2}+4 w^{\prime}-w^{4}-4 z w^{3}-4\left(z^{2}-\alpha\right) w^{2}+4=0 \quad(\gamma=-1)
$$

and the binomial differential equation $y^{\prime 2}=\left(y^{2}-4 \alpha\right)(y-2 z)^{2}$ for $y=w+2 z$. The re-scaling process yields $\mathfrak{y}^{\prime 2}=\mathfrak{y}^{2}(\mathfrak{y}-2)^{2}$ and $\mathfrak{w}^{\prime 2}=\mathfrak{w}^{2}(\mathfrak{w}+2)^{2}$. 


\section{Deficient values and functions}

10.1. The deficiency of zero of fourth transcendents. It is well known and easy to prove that

$$
m\left(r, \frac{1}{w-c}\right)=O(\log r) \quad(c \neq 0)
$$

holds for every Painlevé transcendent. This is also true for $c=0$ except in case of equation (II) with $\alpha=0$ and equation (IV) with $\beta=0$. In [40] it was shown that the Nevanlinna deficiency

$$
\delta(0, w)=\liminf _{r \rightarrow \infty} \frac{m(r, 1 / w)}{T(r, w)}
$$

vanishes for every transcendental solutions to $w^{\prime \prime}=z w+2 w^{3}(\alpha=0)$. In case of equation (IV) it is known that either 0 is a Picard value and $w$ solves some WeberHermite equation $w^{\prime}= \pm\left(2 z w+w^{2}\right)$, or else $\delta(0, w) \leq 1 / 2$ holds (see [11]). The whole truth, however, is more refined and unlikely.

Theorem 10.1. (also [42]) Any transcendental solution to equation (IV) with $\beta=0$ has deficiency $\delta(0, w)=0$, except in the following case: $w$ is sub-normal of order $2 k$ with parameters $\beta=0$ and $\alpha= \pm(2 k+1)$, and

$$
\delta(0, w)=\frac{1}{2 k+1} .
$$

Proof. We first consider the case when $w$ is not sub-normal. Then the simple closed curve $\Gamma_{r}$ that was constructed in Section 2.3 will be used to compute

$$
n_{+}(r, w)-n_{-}(r, w)=\frac{1}{2 \pi i} \int_{\Gamma_{r}} w(z) d z=O\left(r^{2}\right)
$$

here $n_{ \pm}(r, w)$ denotes the number of poles on $|z|<r$ with residue \pm 1 . Then $n_{+}(r, \tilde{w})-$ $n_{-}(r, \tilde{w})=O\left(r^{2}\right)$ also holds for

$$
\tilde{w}=\frac{w^{\prime}-2 z w-w^{2}}{2 w},
$$

and $n(r, 1 / w)=2 n_{+}(r, \tilde{w})$ holds - note that $w$ has only double zeros, $\tilde{w}$ is regular at poles of $w$ with residue -1 and has poles with residue -1 at poles of $w$ with residue 1 . This implies $n(r, 1 / w)=2 n_{-}(r, \tilde{w})+O\left(r^{2}\right)=2 n_{+}(r, w)+O\left(r^{2}\right)=n(r, w)+O\left(r^{2}\right)$ and $N(r, 1 / w)=N(r, w)+O\left(r^{2}\right)=T(r, w)+O\left(r^{2}\right)$. Thus $\lim \sup _{r \rightarrow \infty} T(r, w) / r^{2}=\infty$ implies $\delta(0, w)=0$. It remains to consider sub-normal solutions to equation (IV) with signature $\left[\alpha_{0}^{0} \alpha\right]$ (generic case); the exceptional case $\left[\alpha_{0}^{\alpha} \alpha\right]$ is dealt with in the same manner. These solutions have even order $2 k$ and parameter $\alpha=-2 k-1$. Solutions of order 0 have Picard value zero. If, however, the order is $2 k \geq 2$ we again consider $\tilde{w}$ defined by (39), and remind the reader that again the (double) zeros of $w$ are poles of $\tilde{w}$ with residue +1 , poles of $w$ with residue +1 are poles of $\tilde{w}$ with residue -1 , and $\tilde{w}$ is regular at all other points of the plane, including the poles of $w$ with residue -1 . Then $w$ and $\tilde{w}$ have counting functions $n(r, w) \sim 4(2 k+1) r^{2} / 2 \pi$ and $n_{+}(r, \tilde{w}) \sim n_{-}(r, \tilde{w}) \sim 4 k r^{2} / 2 \pi$, respectively, where $\sim$ means up to some term $o\left(r^{2}\right)$. This implies $n(r, 1 / w)=2 n_{+}(r, \tilde{w}) \sim 8 k r^{2} / 2 \pi$ and $\delta(0, w)=1 /(2 k+1)$. 
Remark 10.1. There is a second way to prove $m(r, 1 / w) \sim 2 r^{2} / \pi$ for $w$ with signature $\left[\alpha_{0}^{0} \alpha\right]$ as follows. Proceeding like in the proof of Theorem 6.1 with $\gamma=0$, $w_{1}=w, w_{2}=0$, and $y=z^{1 / 2}\left(w_{1}-w_{2}\right)=z^{1 / 2} w$ we obtain

$$
y^{\prime \prime}-\left(4 z^{2}-4 \alpha-2+O\left(|z|^{-2}\right)\right) y=0
$$

on the Stokes sectors $\Sigma_{1}$ and $\Sigma_{2}$, hence $-\log |w(z)|=-\operatorname{Re} z^{2}+o\left(|z|^{2}\right)$ on these sectors, while $-\log |w(z)|=O(\log |z|)$ holds on $\Sigma_{0}$ and $\Sigma_{2}$. This gives the assertion, details are left to the reader.

10.2. Deficient rational functions of fourth transcendents. Suppose that $w$ denotes any meromorphic non-rational solution to some algebraic differential equation

$$
\Omega\left(z, w, w^{\prime}, \ldots, w^{(n)}\right)=0 \quad(\Omega \text { some polynomial }),
$$

and let $\phi$ be any rational function such that $\Omega\left(z, \phi, \phi^{\prime}, \ldots, \phi^{(n)}\right) \not \equiv 0$. Then

$$
m\left(r, \frac{1}{w-\phi}\right)=S(r, w),
$$

and the right hand side is $O(\log r)$ if $w$ has finite order of growth. This is a special case of a theorem of Mokhonko-Mokhonko [23], which was used before by Wittich (see his book [46], for example) in many particular cases; if $\phi$ is not rational, the term $O(T(r, \phi))+S(r, \phi)$ has to be added on the right hand side.

To compute $\delta(\phi, w)$ for sub-normal fourth transcendents $w$ and rational functions it suffices to consider solutions $\phi$ to the same equation. Let $\tilde{w}=B[w]$ be any Bäcklund transform of $w$. For definiteness we choose

$$
B[w]=\frac{w^{\prime}-2 \gamma-2 z w-w^{2}}{2 w}
$$

and set $\tilde{\phi}=B[\phi]$. Then $2 w \tilde{w}-2 \phi \tilde{\phi}=w^{\prime}-\phi^{\prime}-2 z(w-\phi)-\left(w^{2}-\phi^{2}\right)$, hence

$$
2(w-\phi) \tilde{w}+2 \phi(\tilde{w}-\tilde{\phi})=w^{\prime}-\phi^{\prime}-(w-\phi)(2 z+w+\phi)
$$

holds, and dividing by $w-\phi$ yields $2 \phi \frac{\tilde{w}-\tilde{\phi}}{w-\phi}=-2 \tilde{w}+\frac{w^{\prime}-\phi^{\prime}}{w-\phi}-2 z-w-\phi$. By the usual rules we thus obtain $m\left(r, \frac{\tilde{w}-\tilde{\phi}}{w-\phi}\right)=O(\log r)$, and $m\left(r, \frac{w-\phi}{\tilde{w}-\tilde{\phi}}\right)=O(\log r)$ by symmetry, thus

$$
m\left(r, \frac{1}{w-\phi}\right)=m\left(r, \frac{1}{\tilde{w}-\tilde{\phi}}\right)+O(\log r) .
$$

The argument works as well for any other and also for iterated Bäcklund transformations. Now every rational solution $\phi(z)=-2 z+\cdots$ and $\phi(z)=\frac{\gamma}{z}+\cdots$ has its origin in $\phi_{0}(z)=0$, that is, there exists some (iterated) Bäcklund transformation, again denoted $B$, such that $B[\phi] \equiv 0$. If $w$ is not sub-normal this yields

$$
m\left(r, \frac{1}{w-\phi}\right)=m\left(r, \frac{1}{B[w]}\right)+O(\log r)=o(T(r, w)),
$$

at least on some sequence $r=r_{n} \rightarrow \infty$. If, however, $w$ is sub-normal of order $k$ we take $B$ such that $\tilde{w}=B[w]$ satisfies some Weber-Hermite equation, which then is also solved by $\tilde{\phi}=B[\phi]$. Then $\tilde{w}-\tilde{\phi}$ has Picard value zero, hence

$$
m\left(r, \frac{1}{w-\phi}\right)=m\left(r, \frac{1}{\tilde{w}-\tilde{\phi}}\right)+O(\log r)=T(r, \tilde{w})+o\left(r^{2}\right)=4 \frac{r^{2}}{2 \pi}+o\left(r^{2}\right)
$$


and $T(r, w)=4(k+1) \frac{r^{2}}{2 \pi}+o\left(r^{2}\right)$ hold in the generic case, this implying $\Delta(\phi, w)=$ $\delta(\phi, w)=1 /(k+1)$. In the exceptional cases the factor 4 has to be replaced by 2 , with the same result. We note that (41) remains true if $w$ is not sub-normal, the rational function $\phi$ is replaced by any sub-normal solution $\phi$, and the term $O(\log r)$ is replaced by $O\left(r^{2}\right)$. We thus have proved

Theorem 10.2. Let $w$ be any fourth transcendent and $\phi$ be any sub-normal or rational solution (but $\phi(z) \not \equiv-\frac{2}{3} z+\cdots$ ) to the very same equation. Then

$$
\delta(\phi, w)= \begin{cases}0 & (w \text { not sub-normal }) \\ \frac{1}{k+1} & (w \text { sub-normal of order } k \geq 1 \text { and } \phi \text { rational }) .\end{cases}
$$

Example 10.1. The sub-normal solutions to equation (IV) with $\alpha=\beta=-2$ have order 1 , solve $w^{\prime 2}+4 w^{\prime}-w^{4}-4 z w^{3}-4\left(z^{2}+2\right) w^{2}+4=0$, and have deficiency $\delta\left(\frac{1}{z}, w\right)=\frac{1}{2}$.

Remark 10.2. Shimomura [29] proved $\delta(\phi, w) \leq 1 / 2$ if $\beta \neq 0$ and $\delta(\phi, w) \leq$ $3 / 4$ if $\beta=0$ for fourth transcendents $w$ and so-called 'small' functions satisfying $T(r, \phi)=S(r, w)$, with the obvious exception that $w$ solves some Weber-Hermite equation.

Remark 10.3. Rational solutions $\phi(z)=-\frac{2}{3} z+\cdots$ arise for parameters $\beta=$ $-2\left(2 n+\frac{1}{3}-\alpha\right)^{2}(n \in \mathbf{Z}), \alpha \in \mathbf{Z}$, and are not related to Weber-Hermite solutions; the most simple case is $\phi(z)=-\frac{2}{3} z, \alpha=0, \beta=-\frac{2}{9}$. The first part of the proof also works in the present case and again yields (40). Thus the general case may be reduced to solutions $w$ to

$$
2 w w^{\prime \prime}=w^{2}+3 w^{4}+8 z w^{3}+4 z^{2} w^{2}-\frac{4}{9} \quad \text { and } \quad \phi(z)=-\frac{2}{3} z,
$$

( $\alpha=0, \beta=-2 / 9)$, but nevertheless requirers a new idea.

10.3. Second transcendents. Theorem 10.1 and 10.2 have an analog for second transcendents. Here rational and sub-normal solutions are separated from each other since they correspond to parameters $\alpha \in \mathbf{Z}$ and $\alpha \in \frac{1}{2}+\mathbf{Z}$, respectively.

Theorem 10.3. Let $w$ be any normal second transcendent. Then

$$
m\left(r, \frac{1}{w-\phi}\right)=O\left(r^{3 / 2}\right)=o(T(r, w))
$$

holds for every meromorphic function satisfying $T(r, \phi)=O\left(r^{3 / 2}\right)$.

Proof. We note that for $\alpha=0$,

$$
m\left(r, \frac{1}{w}\right)=O(\sqrt{T(r, w)})=O\left(r^{3 / 2}\right)
$$

has been proved in [40]. To prove the general result we may assume that $w$ and $\phi$ satisfy one and the same Painlevé equation (II). Then either $\alpha \in \mathbf{Z}$ and $\phi$ is rational or else $\alpha \in \frac{1}{2}+\mathbf{Z}$ and $\phi$ is sub-normal. This time the Bäcklund transformations (34) and the special transformation

$$
z=-\sqrt[3]{2} t, \quad-\sqrt[3]{2} y(t)^{2}=w^{\prime}(z)-w(z)^{2}-z / 2 \quad(\alpha=1 / 2)
$$

play an important role; the latter transforms $w^{\prime \prime}=1 / 2+z w+2 w^{3}$ into $\ddot{y}=t y+2 y^{3}$, hence forms the bridge between $\alpha=1 / 2$ and $\alpha=0$, see [11, p. 142]. 
Consider $\tilde{w}=B[w]=-w+\frac{\alpha-1 / 2}{w^{\prime}-w^{2}-z / 2}$ with $\alpha \neq 1 / 2$, say. Then from

$$
m\left(r, \frac{1}{w^{\prime}-w^{2}-z / 2}\right)=m(r, \tilde{w}+w)+O(1)=O(\log r)
$$

the same estimate with $w$ and $\tilde{w}$ replaced by $\phi$ and $\tilde{\phi}=B[\phi]$, and

$$
\tilde{w}-\tilde{\phi}=-(w-\phi)+(\alpha-1 / 2) \frac{\phi^{\prime}-w^{\prime}+(w-\phi)(w+\phi)}{\left(w^{\prime}-w^{2}-z / 2\right)\left(\phi^{\prime}-\phi^{2}-z / 2\right)}
$$

it follows that $m\left(r, \frac{\tilde{w}-\tilde{\phi}}{w-\phi}\right)=O(\log r)$, hence (40) again holds for every (iterated) Bäcklund transform $\tilde{w}=B[w]$. Like in the proof of Theorem 10.2 this enables us to reduce the general case $\alpha \in \mathbf{Z}$ to the case $\alpha=0$, hence the assertion follows from the corresponding estimate (42). If, however, $\alpha \in \frac{1}{2}+\mathbf{Z}$ we may assume $\alpha=\frac{1}{2}$ and $\phi^{\prime}=\phi^{2}+z / 2$, and use (43): Set $v=w^{\prime}-w^{2}-z / 2$; then the assertion follows from $v=w^{\prime}-\phi^{\prime}-(w-\phi)(w+\phi)$, hence

$$
\frac{1}{w-\phi}=\frac{1}{v}\left(\frac{w^{\prime}-\phi^{\prime}}{w-\phi}-w-\phi\right)
$$

and $m(r, 1 / v)=O(\sqrt{T(r, v)})=O\left(r^{3 / 2}\right)$.

\section{Appendix A: The Phragmén-Lindelöf principle}

The Phragmén-Lindelöf principle is an easy consequence of the two-constantstheorem and may be stated as follows (see also Titchmarsh [43, p. 176-180]):

Theorem 11.1. Let $f$ be a holomorphic function of finite order

$$
\limsup _{z \rightarrow \infty} \frac{\log ^{+} \log ^{+}|f(z)|}{\log |z|}<\frac{\pi}{2 \delta} \quad \text { on } \Sigma:|\arg z| \leq \delta,|z| \geq r_{0} .
$$

Then $f$ is bounded on $\Sigma$ if $f$ is bounded on $\partial \Sigma \backslash\{\infty\}$, and the following is true:

1. If $f$ tends to $c$ as $z=r e^{i \delta} \rightarrow \infty$, then $f$ tends to $c$ as $z \rightarrow \infty$, uniformly on every sector $-\delta+\epsilon<\arg z \leq \delta(0<\epsilon<\delta$ arbitrary $)$.

2. If $f$ tends to $c_{ \pm}$as $z=r e^{ \pm i \delta} \rightarrow \infty$, then $c_{+}=c_{-}=c$, and $f$ tends to $c$ as $z \rightarrow \infty$, uniformly on $|\arg z| \leq \delta$.

Corollary 11.1. Let $h$ be any holomorphic function of finite order on $|\arg z| \leq$ $\eta,|z| \geq r_{0}$, and assume that for every $\delta, 0<\delta \leq \eta$, $h$ satisfies

$$
h(z)=\left\{\begin{array}{ll}
C_{+} e^{a z} z^{\lambda}(1+o(1)) & \left(z=r e^{i \delta} \rightarrow \infty\right) \\
C_{-} e^{b z} z^{\mu}(1+o(1)) & \left(z=r e^{-i \delta} \rightarrow \infty\right)
\end{array} \quad \text { with } C_{+} C_{-} \neq 0 .\right.
$$

Then the following is true:

1. $\operatorname{Re} a=\operatorname{Re} b$ and $\operatorname{Im} a \leq \operatorname{Im} b$.

2. $a=b$ implies $\lambda=\mu$ and $h(z)=C e^{a z} z^{\lambda}(1+o(1))$ as $z \rightarrow \infty$, uniformly on $|\arg z| \leq \eta$; in particular, $h$ has only finitely many zeros.

Proof. In order to prove 1 . we assume $\operatorname{Re} a \neq \operatorname{Re} b$ and even $\operatorname{Re} a>\operatorname{Re} b$ (otherwise take $\overline{h(\bar{z})}$ instead of $h$ to replace $a, b$ by $\bar{b}, \bar{a})$, and consider the holomorphic function

$$
f(z)=e^{-a z} z^{-\lambda} h(z)
$$


it has finite order on $|\arg z| \leq \eta$ and satisfies $f\left(r e^{i \delta}\right) \rightarrow C_{+} \neq 0$ and

$$
\left|f\left(r e^{-i \delta}\right)\right|=O\left(r^{\operatorname{Re}(\mu-\lambda)}\right) e^{\operatorname{Re}(b-a) r \cos \delta+\operatorname{Im}(b-a) r \sin \delta}
$$

as $r \rightarrow \infty$. The right hand side of (45) tends to zero if $\delta>0$ is chosen sufficiently small, hence $f$ is bounded on $|z| \geq r_{0},|\arg z| \leq \delta$ by the Phragmén-Lindelöf principle. The second part of the Phragmén-Lindelöf Principle then gives a contradiction with $c_{+}=C_{+} \neq 0$ and $c_{-}=0$. In the same way obtain a contradiction if we assume $\operatorname{Re} a=\operatorname{Re} b$ and $\operatorname{Im} a>\operatorname{Im} b: f$ tends to $C_{+}$as $z=r e^{i \delta} \rightarrow \infty$, while (45) again implies that $f\left(r e^{-i \delta}\right) \rightarrow 0$ as $r \rightarrow \infty$. This proves $\operatorname{Im} a \leq \operatorname{Im} b$.

In order to prove 2 . we assume $a=b$ and $\operatorname{Re} \lambda>\operatorname{Re} \mu$. Then $f$, again defined by (44), tends to $C_{+} \neq 0$ as $z=r e^{i \delta} \rightarrow \infty$, while $\left|f\left(r e^{-i \delta}\right)\right|=O\left(r^{\operatorname{Re}(\mu-\lambda)}\right)$ tends to zero as $r \rightarrow \infty$. This proves $\operatorname{Re} \lambda \leq \operatorname{Re} \mu$, and in the same way we obtain $\operatorname{Re} \lambda \geq$ $\operatorname{Re} \mu$, hence $\operatorname{Re} \lambda=\operatorname{Re} \mu$. This eventually implies that $f$ tends to $C_{+}$as $z \rightarrow \infty$ on $-\delta+\epsilon<\arg z \leq \delta$ by the Phragmén-Lindelöf principle, hence, in particular, $h(x)=C_{+} e^{a x} x^{\lambda}(1+o(1))$ holds as $x \rightarrow+\infty$ ( $x$ real $)$. In the same way we obtain $h(x)=C_{-} e^{a x} x^{\mu}(1+o(1))$, hence $\lambda=\mu, C_{+}=C_{-}=C$, and $h(z)=C e^{a z} z^{\lambda}(1+o(1))$ holds as $z \rightarrow \infty$ on $|\arg z| \leq \eta$.

\section{Appendix B: Asymptotic expansions}

12.1. Asymptotic expansions of specific solutions. The following theorem on the existence of asymptotic expansions of solutions to algebraic differential equations applies in many different situations to specific solutions.

Theorem 12.1. Let $w$ be any solution to the algebraic differential equation

$$
Q[w]=P(z, w)
$$

satisfying $w(z) \rightarrow c_{0}$ as $z \rightarrow \infty$ on some sector $S$, and assume that

a. $P(z, w)$ is a polynomial in $w$ and rational in $z$ satisfying

$$
P\left(z, c_{0}\right) \rightarrow 0 \quad \text { and } \quad P_{w}\left(z, c_{0}\right) \rightarrow c \neq 0 \quad(z \rightarrow \infty \text { on } S) ;
$$

b. $Q[w]=\sum_{M} a_{M}(z) w^{\ell_{0}} M[w]$ is a differential polynomial with rational coefficients $a_{M}(z)=A_{M} z^{\alpha_{M}}(1+o(1))$ as $z \rightarrow \infty\left(A_{M} \neq 0, \alpha_{M} \in \mathbf{Z}\right)$, and monomials $M[w]=w^{\ell_{1}} \cdots w^{(m)^{\ell_{m}}}$ of weight

$$
d_{M}=2 \ell_{1}+\cdots+(m+1) \ell_{m} \geq \alpha_{M}+2 .
$$

Then $w$ has an asymptotic expansion $w \sim \sum_{k=0}^{\infty} c_{k} z^{-k}$ on $S$.

Proof. We will start with

$$
w(z)=\sum_{\nu=0}^{n} c_{\nu} z^{-\nu}+o\left(|z|^{-n}\right)=\psi_{n}(z)+o\left(|z|^{-n}\right),
$$

which is true for $n=0$. To proceed further we need

$$
w^{\ell_{0}} M[w]=\psi_{n}^{\ell_{0}} M\left[\psi_{n}\right]+o\left(|z|^{-d_{M}-n+1}\right) \quad(z \rightarrow \infty) ;
$$

the proof will be given below. Using (47) we obtain

$$
Q[w]=Q\left[\psi_{n}\right]+o\left(|z|^{\max _{M}\left(\alpha_{M}-d_{M}\right)-n+1}\right)=Q\left[\psi_{n}\right]+o\left(|z|^{-n-1}\right),
$$

hence $w$ satisfies

$$
P(z, w)=Q\left[\psi_{n}\right]+o\left(|z|^{-n-1}\right)
$$


It follows from the first hypothesis that for any rational function $R$ that tends to zero as $z \rightarrow \infty$, the algebraic equation $P(z, y)=R(z)$ has a unique solution that tends to $c_{0}$ as $z \rightarrow \infty$. In particular, equation

$$
P(z, y)=Q\left[\psi_{n}\right](z)
$$

has a unique solution $y_{n}(z)=\sum_{\nu=0}^{\infty} a_{\nu}^{[n]} z^{-\nu}$ about $z=\infty$, and from (48), (49), and

$$
P(z, w(z))-P\left(z, y_{n}(z)\right)=\int_{y_{n}(z)}^{w(z)} P_{\zeta}(z, \zeta) d \zeta=(c+o(1))\left(w(z)-y_{n}(z)\right)
$$

as $z \rightarrow \infty$ (we integrate along the straight line in $\left|\zeta-c_{0}\right|<\delta$ from $y_{n}(z)$ to $w(z)$ ) it follows that

$$
w(z)-y_{n}(z)=o\left(|z|^{-n-1}\right)
$$

hence (46) holds with $n$ replaced by $n+1$ and $\psi_{n+1}(z)=\sum_{\nu=0}^{n+1} a_{\nu}^{[n]} z^{-\nu}$; we note that $a_{\nu}^{[k]}=c_{\nu}(0 \leq \nu \leq k \leq n)$ holds, while the new coefficient is $c_{n+1}=a_{n+1}^{[n]}$.

To prove (47) we first consider the case $\psi_{n}(z) \equiv c_{0}$, hence $M\left[\psi_{n}\right]=0, w^{(k)}(z)=$ $\psi_{n}^{(k)}(z)+o\left(|z|^{-n-k}\right)=o\left(|z|^{-n-k}\right)$, and

$$
w^{\ell_{0}} M[w]=o\left(|z|^{\ell_{1}(-n-1)+\cdots+l_{m}(-n-m)}\right)=o\left(|z|^{-d_{M}-n+1}\right)
$$

since $-\sum_{k=1}^{m}(n+k) \ell_{k} \leq-d_{M}-(n-1) \sum_{k=1}^{m} \ell_{k} \leq-d_{M}-n+1$. Thus (47) holds with $M\left[\psi_{n}\right] \equiv 0$.

Otherwise let $c_{\nu}$ be the first non-zero coefficient of $\psi_{n}$ with index $\nu \geq 1$. Then

$$
\begin{aligned}
\psi_{n}^{(k)}(z) & =O\left(|z|^{-\nu-k}\right), \\
w^{(k)}(z) & =\psi_{n}^{(k)}(z)+o\left(|z|^{-n-k}\right)=O\left(|z|^{-\nu-k}\right), \\
\left(w^{(k)}(z)\right)^{\ell_{k}} & =\left(\psi_{n}^{(k)}(z)\right)^{\ell_{k}}+O\left(|z|^{-(\nu+k)\left(\ell_{k}-1\right)}\right) o\left(|z|^{-n-k}\right) \\
& =\left(\psi_{n}^{(k)}(z)\right)^{\ell_{k}}+o\left(|z|^{-k \ell_{k}-n-\nu\left(\ell_{k}-1\right)}\right)
\end{aligned}
$$

and $M[w]=M\left[\psi_{n}\right]+R_{n}$ holds with remainder term

$$
\begin{aligned}
R_{n} & =\sum_{\ell_{j}>0} \prod_{k \neq j}\left(\psi_{n}^{(k)}(z)\right)^{\ell_{k}} o\left(|z|^{-j \ell_{j}-n-\nu\left(\ell_{j}-1\right)}\right) \\
& =\sum_{\ell_{j}>0} o\left(|z|^{-\sum_{k \neq j} \ell_{k}(k+\nu)-j \ell_{j}-n-\nu\left(\ell_{j}-1\right)}\right)=o\left(|z|^{-d_{M}-n+1}\right),
\end{aligned}
$$

since $\nu-\sum_{k=1}^{m} \ell_{k}(\nu-1) \leq 1$, hence

$$
\begin{aligned}
w^{\ell_{0}} M[w] & =\psi_{n}^{\ell_{0}} M\left[\psi_{n}\right]+o\left(|z|^{-n}\right) M\left[\psi_{n}\right]+o\left(|z|^{-d_{M}-n+1}\right) \\
& =\psi_{n}^{\ell_{0}} M\left[\psi_{n}\right]+o\left(|z|^{-n-\sum_{k=1}^{m}(k+\nu) \ell_{k}}\right)+o\left(|z|^{-d_{M}-n+1}\right) \\
& =\psi_{n}^{\ell_{0}} M\left[\psi_{n}\right]+o\left(|z|^{-d_{M}-n+1}\right) .
\end{aligned}
$$

12.2. Existence of solutions with specific asymptotic expansions. Theorem 12.1 says that some specific solution has an asymptotic expansion. This may not be mixed up with the well-known and in some sense much more general Theorems 12.1 and 14.1 in Wasow [44], which assert the existence of some solution having an asymptotic expansion. To the convenience of the reader we will prove existence of first, second, and fourth Painlevé transcendents with prescribed asymptotic expansions according to (19) on arbitrary sectors $S$ with central angle $\Theta=\frac{4}{5} \pi, \Theta=\frac{2}{3} \pi$, and $\Theta=\frac{1}{2} \pi$, respectively. 
Theorem 12.2. To every such sector $S$ there exists some first, second, and fourth Painlevé transcendent with prescribed asymptotic expansion (19)(i), (ii), and (iv) on $S$, respectively.

Proof. According to our philosophy we prefer to consider equation (IV) in detail. We set $t=z^{2}$ and $w(z)=t^{-1 / 2} v(t)^{2}$ to obtain

$$
\ddot{v}=\frac{v^{4}-\gamma^{2}}{4 v^{3}}+\frac{1}{4 t}\left(2 v^{3}-\alpha v\right)+\frac{3}{16 t^{2}}\left(v^{5}-v\right)=f(t, v) \quad\left(\gamma^{2}=-\beta / 2 \neq 0\right),
$$

hence $\dot{\mathfrak{x}}=\mathfrak{f}(t, \mathfrak{x})$ when written as a system, with $\mathfrak{x}=\left(\begin{array}{l}x \\ y\end{array}\right), x=v-\sqrt{\gamma}, y=\dot{v}$, $\mathfrak{f}(t, \mathfrak{x})=\left(\begin{array}{c}y \\ f(t, x+\sqrt{ \pm \gamma})\end{array}\right)$, and Jacobian $\lim _{t \rightarrow \infty} \mathfrak{f}^{\prime}(t, \mathfrak{x})=\left(\begin{array}{ll}0 & 1 \\ 1 & 0\end{array}\right)$. From the latter it follows that there exists a unique formal solution $\sum_{k=1}^{\infty} \mathfrak{x}_{k} t^{-k}$, hence a solution with asymptotic expansion $\mathfrak{x}(t) \sim \sum_{k=1}^{\infty} \mathfrak{x}_{k} t^{-k}$ on any given sector of central angle $\pi$, which itself gives rise to a solution to (IV) with asymptotic expansion (19)(iv) ${ }_{c}^{ \pm}$on any given sector of central angle $\pi / 2$. Similarly, the substitution $t=z^{2}, w(z)=t^{1 / 2} v(t)^{2}$ yields

$$
\ddot{v}+\frac{\dot{v}}{t}=\frac{1}{16} v\left(v^{2}+2\right)\left(3 v^{2}+2\right)-\frac{\alpha v}{4 t}+\frac{1}{16 t^{2}}\left(v-\frac{4 \gamma^{2}}{v^{3}}\right),
$$

hence we obtain solutions having asymptotic expansions (iv) $a$ and (iv) ${ }_{b}$ (according to $\lim _{t \rightarrow \infty} v(t)^{2}=-2 / 3$ and $\lim _{t \rightarrow \infty} v(t)^{2}=-2$ ), respectively. Again the sector $S$ in the $z$-plane with central angle $\pi / 2$ may be prescribed. In the other cases we just note the substitutions and differential equations:

$$
\begin{array}{lll}
\text { (i) } \quad z=t^{4 / 5}, w(z)=t^{2 / 5} v(t): & \ddot{v}+\frac{\dot{v}}{t}=\frac{96}{25}\left(6 v^{2}+1\right)+\frac{4 v}{25 t^{2}} . \\
\text { (ii) })_{a} & z=t^{2 / 3}, w(z)=t^{-2 / 3} v(t): & \ddot{v}-\frac{\dot{v}}{t}=\frac{4}{9}(v+\alpha)+\frac{8}{9 t^{2}}\left(v^{3}-v\right) . \\
\text { (ii) })_{b} & z=t^{2 / 3}, w(z)=t^{1 / 3} v(t): & \ddot{v}+\frac{\dot{v}}{t}=\frac{4}{9}\left(v+2 v^{3}\right)+\frac{4 \alpha}{9 t}+\frac{v}{9 t^{2}} .
\end{array}
$$

\section{References}

[1] Ablowitz, M., and H. Segur: Asymptotic solutions of the Korteweg-de Vries equation. Stud. Appl. Math. 57, 1977, 13-44.

[2] Ablowitz, M., and H. Segur: Exact linearization of a Painlevé transcendent. - Phys. Rev. Lett. 38, 1977, 1103-1106.

[3] Bassom, A., P. Clarkson, and A. Hicks: Bäcklund transformations and solution hierarchies for the fourth Painlevée equation. - Stud. Appl. Math. 95, 1995, 1-71.

[4] Boutroux, P.: Recherches sur les transcendentes de M. Painlevé et l'étude asymptotique des équations différentielles du seconde ordre. - Ann. École Norm. Supér. 30, 1913, 255-375, and 31, 1914, 99-159.

[5] Chuang, C.-T.: Normal families of meromorphic functions. - World Scientific, 1993.

[6] Clarkson, P., and J. McLeod: Integral equations and connection formulae for the Painlevé equations. - In: Painlevé Transcendents, their Asymptotics and Physical Applications 131, ed. P. Winternitz and D. Levi, New York, 1992.

[7] Classen, C.: Subnormale Lösungen der vierten Painlevéschen Differentialgleichung. - PhD thesis, Dortmund, 2015, 1-78.

[8] Eremenko, A.: Meromorphic solutions of algebraic differential equations. - Russian Math. Surveys 37, 1982, 61-95.

[9] Eremenko, A.: Meromorphic solutions first-order of algebraic differential equations. - Funct. Anal. Appl. 18, 1984, 246-248. 
[10] Fokas, A., A. Its, A. Kapaev, and V. Novokshënov. - Painlevé transcendents. The Riemann-Hilbert approach. - Math. Surveys Monogr. 128, 2006.

[11] Gromak, V., I. Laine, and S. Shimomura: Painlevé differential equations in the complex plane. - W. de Gruyter, Berlin-New York, 2002.

[12] Hastings, S., and J. McLeod: A boundary value problem associated with the second Painlevée transcendent and the Korteweg-de Vries equation. - Arch. Ration. Mech. Anal. 73, $1980,31-51$.

[13] Hayman, W.: Meromorphic functions. - Oxford Clarendon Press, 1964.

[14] Hille, E.: Ordinary differential equations in the complex domain. - Dover Publ., 1997.

[15] Hinkmanen, A., and I. Laine: Solutions of the first and second Painlevé equations are meromorphic. - J. Anal. Math. 79, 1999, 345-377.

[16] Hinkkanen, A., and I. Laine: Growth results for Painlevé transcendents. - Math. Proc. Camb. Phil. Soc. 137, 2004, 645-655.

[17] Hinkmanen, A., and I. Laine: Order of growth of Painlevé transcendents. In: Complex Analysis and Applications, World Scientific, 2006, 72-77.

[18] Hinkmanen, A., and I. Laine: Growth of second Painlevé transcendents. - Preprint, 2012.

[19] Its, A., and A. KAPAEv: Connection formulae for the fourth Painlevé transcendent; ClarksonMcLeod solution. - J. Phys. A: Math. Gen. 31, 1998, 4073-4113.

[20] Joshi, N., and A. Kitaev: On Boutroux's tritonqée solutions of the first Painlevé equation. - Stud. Appl. Math. 107, 2001, 253-291.

[21] Kitaev, A. V.: Elliptic asymptotics of the first and the second Painlevé transcendents. Russian Math. Surveys 49, 1994, 81-150.

[22] Lukashevich, N.: Theory of the fourth Painlevé equation. - Differential Equations 3, 1967, 395-399.

[23] Moknonko, A.Z., and V.D. Moknonko: Estimates for the Nevanlinna characteristics of some classes of meromorphic functions and their applications to differential equations. - Siberian Math. J. 15, 1974, 921-934.

[24] Mues, E., and R. Redheffer: On the growth of logarithmic derivatives. - J. London Math. Soc. 8, 1974, 412-425.

[25] Novokshënovm, V.: Padé approximations of Painlevé I and II transcendents, - Theor. Math. Phys. 159, 2009, 853-862 (in Russian).

[26] Novokshënov, V.: Tronqué solutions of the Painlevé II equation. - Theor. Math. Phys. 172, $2012,1135-1145$.

[27] Schubart, H., und H. Wittich: Zur Wachstumsordnung der Lösungen einer Klasse nichtlinearer Differentialgleichungen. - Arch. Math. 9, 1958, 355-359.

[28] Shimomura, S.: Value distribution of the Painleve transcendents of the first and second kind. - J. Anal. Math. 82, 2000, 333-346.

[29] Shimomura, S.: On deficiencies of small functions for Painlevé transcendents of the fourth kind. - Ann. Acad. Sci. Fenn. Math. 27, 2002, 109-120.

[30] Shimomura, S.: Lower estimates for the growth of Painlevé transcendents. - Funkcial. Ekvac. 46, 2003, 287-295.

[31] Shimomura, S.: Proofs of the Painlevé property for all Painlevé equations. - Jap. Journal Math. 29, 2003, 159-180.

[32] Shimomura, S.: Growth of the first, the second and the fourth Painleve transcendents. Math. Proc. Camb. Phil. Soc. 134, 2003, 259-269.

[33] Shimomura, S.: Lower estimates for the growth of the fourth and the second Painleve transcendents. - Proc. Edinb. Math. Soc. 47, 2004, 231-249. 
[34] SRIPonpaew, B.: The order of growth of the fourth Painlevé transcendents. - PhD thesis, Univ. of Illinois at Urbana-Champaign, 2012.

[35] Steinmetz, N.: Rational iteration. Complex analytic dynamical systems. - de Gruyter, 1993.

[36] Steinmetz, N.: On Painlevé's equations I, II and IV. - J. Anal. Math. 82, 2000, 363-377.

[37] Steinmetz, N.: Value distribution of the Painlevé transcendents. - Israel J. Math. 128, 2002, $29-52$.

[38] Steinmetz, N.: Boutroux's method vs. re-scaling. Lower estimates for the orders of growth of the second and fourth Painlevé transcendents. - Port. Math. 61, 2004, 369-374.

[39] Steinmetz, N.: The Yosida class is universal. - J. Anal. Math. 117, 2012, 347-364.

[40] Steinmetz, N.: Sub-normal solutions to Painlevé's second differential equation. - Bull. London Math. Soc. 45, 2013, 225-235.

[41] Steinmetz, N.: Complex Riccati differential equations revisited. - Ann. Acad. Sci. Fenn. Math. 39, 2014, 503-511.

[42] Steinmetz, N.: An old new class of meromorphic functions. - J. Anal. Math., 2016 (to appear).

[43] Titchmarsh, E. C.: The theory of functions. - Oxford Univ. Press, 1979.

[44] Wasow, W.: Asymptotic expansions for ordinary differential equations. - J. Wiley \& Sons, 1965.

[45] Wiтtich, H.: Eindeutige Lösungen der Differentialgleichungen $w^{\prime \prime}=P(z, w)$. - Math. Ann. $125,1953,355-365$.

[46] Wiтtich, H.: Neuere Untersuchungen über eindeutige analytische Funktionen. - Springer, 1968.

[47] Yosida, K.: On a class of meromorphic functions. - Proc. Phys. Math. Soc. Japan 16, 1934, $227-235$.

[48] Zalcman, L.: A heuristic principle in function theory. - Amer. Math. Monthly 82, 1975, 813-817.

[49] Zalcman, L.: Normal families: new perspectives. - Bull. Amer. Math. Soc. 35, 1998, 215-230.

Received 26 February 2016 • Accepted 15 April 2016 